\title{
Numerical study of bio-convection flow of magneto- Cross nanofluid containing gyrotactic microorganisms with effective Prandtl number approach
}

\section{Aamir Hamid}

Quaid-i-Azam University

M. ljaz Khan ( $\square$ mikhan@math.qau.edu.pk)

Riphah International University

R. Naveen Kumar

Davangere University

R. J. Punith Gowda

Davangere University

B.C. Prasannakumara

Davangere University

\section{Research Article}

Keywords: Cross nanofluid, MHD, thermal radiation, binary chemical reaction, Bioconvection, Gyrotactic microorganisms

Posted Date: April 23rd, 2021

DOl: https://doi.org/10.21203/rs.3.rs-435508/v1

License: (c) (i) This work is licensed under a Creative Commons Attribution 4.0 International License.

Read Full License

Version of Record: A version of this preprint was published at Scientific Reports on August 6th, 2021. See the published version at https://doi.org/10.1038/s41598-021-95587-2. 


\title{
Numerical study of bio-convection flow of magneto-Cross nanofluid containing gyrotactic microorganisms with effective Prandtl number approach
}

\author{
${ }^{1}$ Aamir Hamid, ${ }^{2}$ M. Ijaz Khan, ${ }^{3}$ R. Naveen Kumar, ${ }^{3}$ R. J. Punith Gowda, ${ }^{3}$ B.C. Prasannakumara \\ ${ }^{1}$ Department of Mathematics, Quaid-i-Azam University, Islamabad 44000 , Pakistan \\ ${ }^{2}$ Department of Mathematics and Statistics, Riphah International University I-14, Islamabad \\ 44000, Pakistan \\ ${ }^{3}$ Department of Studies and Research in Mathematics, Davangere University, Davangere, \\ Karnataka, INDIA
}

("Corresponding author email: mikhan@math.qau.edu.pk (M. I. Khan))

\begin{abstract}
:
In this study, a mathematical model is developed to scrutinize the transient magnetic flow of Cross nanoliquid past a stretching sheet with thermal radiation effects. Binary chemical reactions and heat source/sink effects along with convective boundary condition are also taken into the consideration. Apposite similarity transformations are utilized to transform partial differential equations (PDE's) into ordinary ones and then numerically tackled by shooting method. The Impacts of different emerging parameters on the thermal, concentration, velocity, and microrotation profiles are incorporated and discussed in detail by means of graphs. Results reveals that, the escalation in Magnetic parameter and Rayleigh number slowdowns the velocity and momentum of the fluid. The increase in Biot number, radiation and Heat sink/source parameters upsurges the thermal boundary but, converse trend is seen for escalating Prandtl number. The density number of motile microorganisms acts as a growing function of bioconvection Lewis number and declining function of bioconvection Peclet number.
\end{abstract}

Keywords: Cross nanofluid; MHD; thermal radiation; binary chemical reaction; Bioconvection; Gyrotactic microorganisms.

\section{Introduction}

Researchers and scientists nowadays have more research fields due to developments in 
nanotechnology and nanoscience. Nanofluids are proving to be useful in a variety of situations, including heat transference Technological advances demand efficient heat conveyance processes, and nanoliquids provide a more effective medium for heat passage from one source to the other. In this regard different fluid models have been presented over time to illustrate fluid properties. The Cross-viscosity model for generalized Newtonian fluid was proposed by Cross. By using this concept, Khan et .al [1] elucidated the chemically reacting magnetohydrodynamic (MHD) radiative flow of cross nanoliquid towards a stretchy sheet. Ali et .al [2] explicated the radiative stream of cross nanoliquid instigated by a stretchy sheet. Abbas et .al [3] expounded the MHD convective stream of cross nanoliquid with thermal radiation. Waqas et .al [4] investigated the bioconvective stream of cross nanoliquid flow with radiation and melting effect. Xiong et .al [5] illuminated the chemically reacting dissipative flow of cross nanoliquid with mixed convection.

The studies of magnetohydrodynamic (MHD) streams of non-Newtonian and Newtonian liquids over a stretching surface have gotten a lot of interest because of their wide range of uses in chemical engineering and metallurgy. Such MHD flow investigations are critical in industry and have uses in a number of fields, including petroleum processing and metallurgical processes. The rate of cooling, as well as the desired end product properties, can be regulated by using electrically conducting fluids and applying a magnetic field. In the purification of molten metals from nonmetallic inclusions, a magnetic field has been used. Recently, Hayat et .al [6] elucidated the MHD radiative stream of liquid past a porous stretchy sheet. thermal radiation. Radhika et .al [7] explicated the MHD steam of Dusty nanoliquid instigated by an elastic sheet with melting effect. Senapati et .al [8] expounded the MHD flow of Casson nanoliquid flow above a stretch sheet. Patil et .al [9] deliberated the chemically reacting radiative stream of Powel-Eyring liquid above a stretchy sheet. Naidu et .al [10] elucidated the MHD radiative stream of Jeffrey nanoliquid above a stretchy sheet with Gyrotactic microorganisms.

The mechanism of thermal radiation involves a hot body releasing electromagnetic radiation in both directions. Many objects on the planet emit radiation in the infrared range of the electromagnetic spectrum. Thermal radiation is important in heat nuclear reactor protection, power plants, exchangers, furnace architecture, solar energy, and power technology. Motivated by these uses, Khan et .al [11] explored the radiative flow of Carreau liquid above a stretchy surface. Hashim et .al [12] explicated the radiative stream of Williamson nanoliquid above a stretchy 
geometry. Ali et .al [13] elucidated the radiative flow of nanoliquid with heat production/absorption. Reddy et .al [14] expounded the radiative flow of nanoliquid past a melting surface. Ijaz et .al [15] explicated the effect of radiation and magnetic dipole on bioconvective flow of Jeffery nanofluid.

The relationships between chemical reactions and mass transport are typically complex, as shown by the synthesis and absorption of reactant species at various rates both within the liquid and during mass transfer. Chemical reactions have plentiful usages in fields engineering and sciences like generator electric power and food processing. The concept of binary chemical reaction was initially elucidated by Merkin [16]. Rasool et .al [17] exemplified the impact of binary chemical reaction on Williamson nanoliquid above an elastic sheet. Wang et .al [18] elucidated the chemically reactive dissipative stream of Carreau nanoliquid above a stretchy surface. Gowda et .al [19] expounded the impact of chemical reaction on nanoliquid stream past a stretchy sheet coiled in a circle. Khan et .al [20] expounded the impact of binary chemical reaction on bioconvective stream of nanoliquid.

Bio-convection is a natural process that occurs as microorganisms move randomly in single-celled or colony-like formation. The directional motion of various forms of microorganisms is the basis for various bio-convection systems. Gyrotactic microorganisms are those that swim upstream against gravity in still water, causing the upper portion of the suspension to be denser than the lower part. Bioconvection's importance can be seen in a variety of bio-microsystems, such as biotechnology related to mass transportation, enzyme biosensors and mixing. Recently, Khan et .al [21] elucidated the MHD bioconvective stream of Newtonian fluid with chemical reaction. Chu et .al [22] explicated the MHD bioconvective stream of third grade liquid past a stretchy sheet by using Buongiorno model. Al-Khaled et .al [23] expounded the radiative bioconvective stream of nanoliquid. Jayadevamurthy et .al [24] elucidated the bioconvection flow of hybrid nanofluid instigated by a moving spinning disk. Arafa et .al [25] explicated MHD bioconvective stream of nanofluid with convective boundary constraint and radiation effect.

It is familiar that there are various approaches that could be contemplated in order to explain few realistic solutions for this specific type of issue. However, to the best of the authors' understanding, no numerical solution has been earlier inspected for magnetic flow of Cross nanofluid past a 
stretching sheet with thermal radiation effects. The focal point in the current paper is to examine the above-described flow numerically.

\section{Mathematical formulation}

In this study, we have considered transient two-dimensional magnetic flow of an incompressible Cross nanofluid past a stretching sheet in the presence of thermal radiation effects. A non-uniform time dependent transverse magnetic field of strength $B_{0}$ is applied perpendicular to flow direction (see Fig. 1). Since the magnetic Reynolds number is low, the induced magnetic field can be ignored. The effect of Brownian motion and thermophoresis is incorporated into a currently developed framework for nanofluid. The implementation of a critical practical concentration condition, namely the zero nanoparticles mass flux condition. The convective boundary condition is also taken into consideration. Binary chemical reactions and heat source/sink effects are also taken into consideration. The suspension of nanoparticles in nanofluid is achieved through the use of a surfactant or surface charge technology. This prevents nanoparticles from adhering to the surface and accumulating.

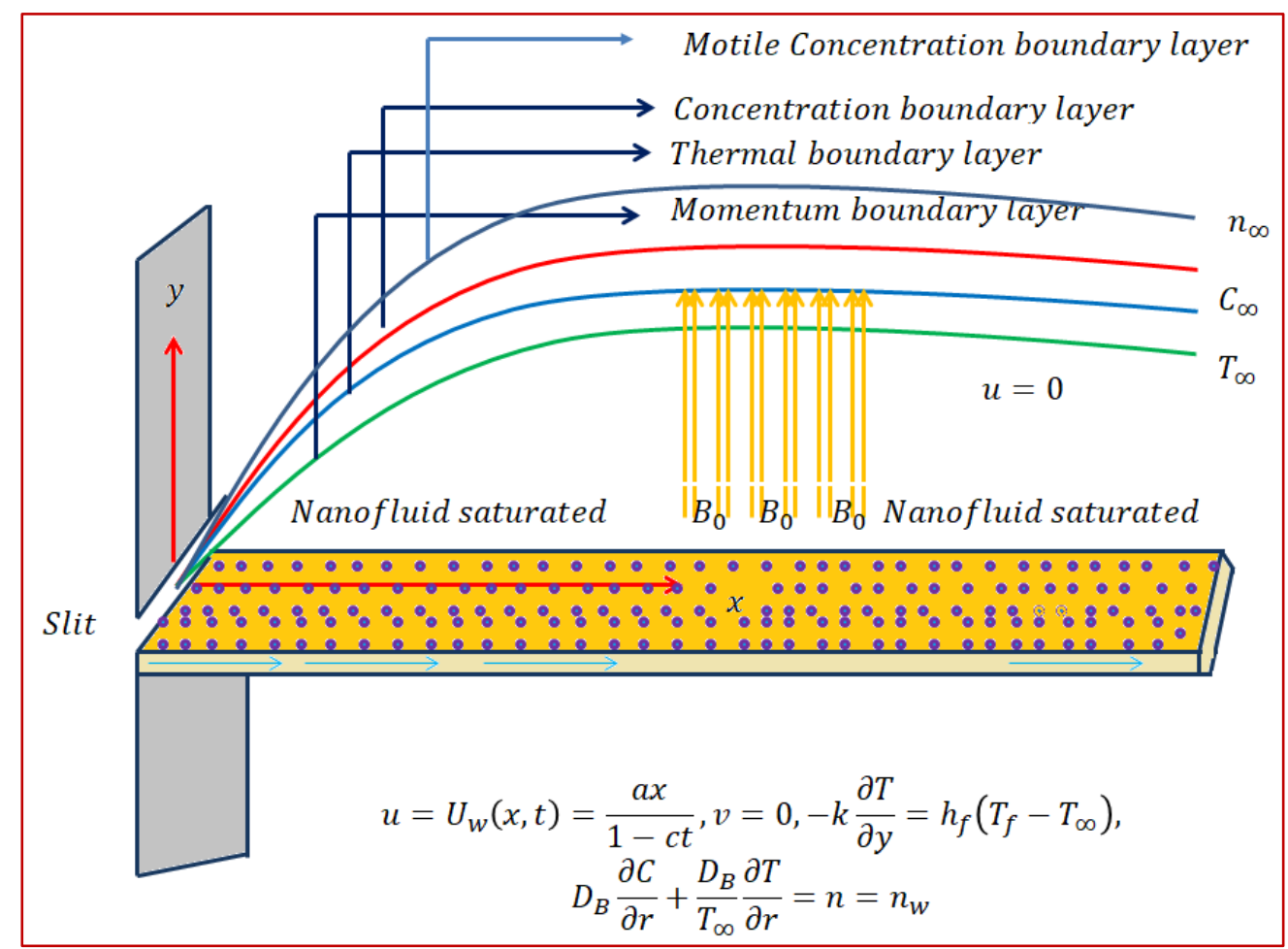

Fig. 1: Schematic sketch of the physical model.

The governing equations of the above described flow are:

\section{Continuity:}


$\frac{\partial u}{\partial x}+\frac{\partial v}{\partial r}=0$

Momentum equation:

$$
\begin{aligned}
\frac{\partial u}{\partial t}+u \frac{\partial u}{\partial x}+v \frac{\partial u}{\partial y}= & v\left[\frac{\frac{\partial u}{\partial y}}{1+\left\{\Gamma\left(\frac{\partial u}{\partial y}\right)^{1-n}\right\}^{2}}\right]-\frac{\sigma B^{2}(t)}{\rho_{f}} u+ \\
& \frac{1}{\rho_{f}}\left[g \rho_{f}\left(T-T_{\infty}\right) \beta\left(1-C_{\infty}\right)-g\left(\rho_{p}-\rho_{f}\right)\left(C-C_{\infty}\right)-g\left(\rho_{m}-\rho_{\infty}\right) \gamma\left(n-n_{\infty}\right)\right] .
\end{aligned}
$$

Energy equation:

$$
\begin{array}{r}
\frac{\partial T}{\partial t}+u \frac{\partial T}{\partial x}+v \frac{\partial T}{\partial y}=\left[\alpha_{f}+\frac{16 \sigma^{*} T_{\infty}^{3}}{3 k^{*}\left(\rho c_{f}\right)} \frac{\partial^{2} T}{\partial y^{2}}\right]+\tau\left[D_{B} \frac{\partial C}{\partial r} \frac{\partial T}{\partial r}+\frac{D_{T}}{T_{\infty}}\left(\frac{\partial T}{\partial r}\right)^{2}\right]+ \\
\frac{Q_{0}}{\left(\rho c_{p}\right)_{f}}[(T-T)] .
\end{array}
$$

\section{Concentration equation:}

$$
\begin{array}{r}
\frac{\partial C}{\partial t}+u \frac{\partial C}{\partial x}+v \frac{\partial C}{\partial y}=D_{B} \frac{\partial^{2} C}{\partial y^{2}}+\frac{D_{T}}{T_{\infty}}\left(\frac{\partial^{2} C}{\partial y^{2}}\right)- \\
k_{r}^{2}\left(C-C_{\infty}\right)\left(\frac{T}{T_{\infty}}\right)^{n^{*}} \exp \left(-\frac{E_{a}}{k^{*} T}\right) .
\end{array}
$$

Bioconvection equation:

$$
\frac{\partial n}{\partial t}+u \frac{\partial n}{\partial x}+v \frac{\partial n}{\partial y}+\frac{b_{1} W_{c}}{C_{\infty}}\left[\frac{\partial}{\partial y}\left(n \frac{\partial C}{\partial r}\right)\right]=D_{m}\left(\frac{\partial^{2} n}{\partial y^{2}}\right)
$$

The physical realistic boundary conditions are

$$
u=U_{w}(x, t)=\frac{a x}{1-c t}, v=0,-k \frac{\partial T}{\partial y}=h_{f}\left(T_{f}-T_{\infty}\right), D_{B} \frac{\partial C}{\partial r}+\frac{D_{T}}{T_{\infty}} \frac{\partial T}{\partial r}=0, n=n_{w} \text { at } y=0,(\mathbf{6})
$$


$u \rightarrow 0, T \rightarrow T_{\infty}, C \rightarrow C_{\infty}, n \rightarrow n_{\infty}$ as $y \rightarrow \infty$. (7)

We have the similarity transformations

$\eta=y \sqrt{\frac{U_{w}}{v x}}, \psi=\sqrt{v U_{w} x} f(\eta), \theta(\eta)=\frac{T-T_{\infty}}{T_{f}-T_{\infty}}, \phi(\eta)=\frac{C-C_{\infty}}{C_{\infty}}, \chi(\eta)=\frac{n-n_{\infty}}{n_{w}-n_{\infty}} .(\mathbf{8})$

The stream function $\psi(r, x)$ is given by $u=\frac{1}{r} \frac{\partial \psi}{\partial r}$ and $v=-\frac{1}{r} \frac{\partial \psi}{\partial x}$,

By utilizing Eq. (8) in Eqs. (1) - (5) with associated boundary constraints revealed in Eqs. (6) and (7), we came know that Eq. (1) satisfied automatically and Eqs. (2-5) take the following form

$$
\begin{array}{r}
\left(f f^{\prime \prime}-\left(f^{\prime}\right)^{2}\right)\left[1+\left(W e f^{\prime \prime}\right)^{1-n}\right]-M^{2} f^{\prime}\left[1+\left(W e f^{\prime \prime}\right)^{1-n}\right]+\left[1+n\left(W e f^{\prime \prime}\right)^{1-n}\right] f^{\prime \prime \prime} \\
-A\left(f^{\prime}+\frac{\eta}{2} f^{\prime \prime}\right)+\lambda\left(\theta-N_{r} \phi-R_{b} \chi\right)=0, \\
\left(1+\frac{4}{3} R d\right) \frac{1}{\operatorname{Pr}} \theta^{\prime \prime}+f \theta^{\prime}+A \frac{\eta}{2} \theta^{\prime}+N_{b} \theta^{\prime} \phi^{\prime}+N_{t} \theta^{\prime 2}+Q_{0} \theta=0,(\mathbf{1 0}) \\
\phi^{\prime \prime}+S c f \phi^{\prime}+\frac{N_{t}}{N_{b}} \theta^{\prime \prime}+S c A \frac{\eta}{2} \phi^{\prime}-\sigma(1+\delta \theta)^{n^{*}} \phi \exp \left(-\frac{E_{1}}{1+\delta \theta}\right)=0,(\mathbf{1 1}) \\
\chi^{\prime \prime}+L b f \chi^{\prime}-P e\left[\chi^{\prime} \phi^{\prime}+(\chi+\Omega)\right]+L b A \frac{\eta}{2} \chi^{\prime}=0,(\mathbf{1 2})
\end{array}
$$

and transformed boundary conditions are:

$$
f(0)=0, f^{\prime}(0)=1, \theta^{\prime}(\eta)=-B i[1+\theta(\eta)], N_{b} \phi^{\prime}+N_{t} \theta^{\prime}=0, n=n_{w}
$$


$f^{\prime}(\infty) \rightarrow 0, \theta(\infty) \rightarrow 0, \phi(\infty) \rightarrow 0, \chi(\infty) \rightarrow 0$

where prime denotes the derivative with respect to $\{$ and the dimensionless physical parameters are defined as follows:

$M=\frac{\sigma B_{0}^{2}}{2 \rho a}$ the magnetic parameter, $L b=\left(\frac{\alpha}{D_{n}}\right)$ the bioconvection Lewis number, $R d=\frac{16 \sigma^{*} T_{\infty}^{3}}{3 k^{*} k_{f}}$ the radiation parameter and $W e=a \Gamma \operatorname{Re}^{1 / 2}$ the Weissenberg number, $Q_{0}=\left(\frac{Q(1-c t)}{a(\rho c)_{f}}\right)$ the heat generation/absorption parameter, $N b=\left(\frac{\tau D_{B}\left(C_{f}-C_{\infty}\right)}{v}\right)$ the Brownian motion parameter, $A=\left(\frac{c}{a}\right)$ the unsteadiness parameter, $S c=\left(\frac{v}{D_{B}}\right)$ the Schmidt number, $\operatorname{Pr}=\left(\frac{\mu c_{p}}{k}\right)$ the Prandtl number, $P e=\left(\frac{b_{1} W_{c}}{D_{n}}\right)$ the bioconvection Peclet number, $L e=\left(\frac{\alpha}{D_{B}}\right)$ the traditional Lewis number, $N t=\left(\frac{\tau D_{B}\left(T_{f}-T_{\infty}\right)}{V T_{\infty}}\right)$ the thermophoresis parameter, $N_{r}=\left(\frac{\left(\rho_{p}-\rho_{f}\right) \Delta C_{w}}{\rho_{f} \beta\left(1-C_{\infty}\right) \Delta T_{f}}\right)$ the buoyancy ratio parameter, $R b=\left(\frac{\gamma \Delta \rho \Delta n_{w}}{\rho_{f} \beta\left(1-C_{\infty}\right) \Delta T_{f}}\right)$ the bioconvection Rayleigh number, $\sigma=\left(\frac{n_{\infty}}{\Delta n_{w}}\right)$ the bioconvection constant.

It should be remembered that the physical quantities of engineering concern, namely the local skin friction coefficient, local Nusselt quantity, and motile microorganisms, are also essential characteristics of the current investigation.

$$
C_{f}=\frac{\tau_{w}}{\rho U_{w}^{2}}, \quad N u_{x}=\frac{x q_{w}}{k_{f}\left(T_{f}-T_{\infty}\right)}, N n_{x}=\frac{x q_{n}}{D_{n}\left(n_{w}-n_{\infty}\right)}
$$

where $\tau_{w}, q_{w}, q_{n}$ is the wall shear stress and wall heat flux and wall motile microorganism flux, respectively, having the following form:

$$
\tau_{w}=\mu_{0}\left(\frac{\frac{\partial u}{\partial y}}{1+\left\{\Gamma\left(\frac{\partial u}{\partial y}\right)^{1-n}\right\}}\right)_{y=0}, q_{w}=-k\left(\frac{\partial T}{\partial y}\right)_{y=0}, q_{n}=-D_{n}\left(\frac{\partial n}{\partial y}\right)_{y=0} .
$$

On substituting Eq. (16) into Eq. (15), we obtain

$$
\operatorname{Re}^{1 / 2} C_{f}=\frac{2 f^{\prime \prime}(0)}{\left[1+\left\{W e f^{\prime \prime}(0)\right\}^{1-n}\right]}, \operatorname{Re}^{-1 / 2} N u_{x}=-\left(1+\frac{4}{3} R d\right) \theta^{\prime}(0), \operatorname{Re}^{-1 / 2} N n_{x}=-\chi^{\prime}(0),
$$

\section{Discussion of results}

The transient two-dimensional magnetic flow of Cross nanofluid past a stretching sheet with 
convective boundary condition, thermal radiation, binary chemical reactions and heat source/sink is considered in the present model. The Buongiorno model incorporated into a currently developed framework for nanofluid. This section manifests the salient features and the rheological behaviour of various flow physical non-dimensional parameters associated in the dimensionless equations. Consideration is engrossed here to point out physical influence of these parameters on velocity, concentration, thermal and motile gyrotactic profile graphically (Figs. 2-22) by varying one parameter and other parameters are kept constant.

Fig. 2 depicts the flow control of the $M$ on the velocity profile. Here, increase in $M$ slowdowns the velocity and momentum of the fluid. Practically it has been noticed that the existence of a magnetic field in the stream field region decelerates the fluid motion. These findings suggest that magnetization force provides additional struggle to the stream which declines its velocity. Fig. 3 demonstrates the influence of the $W e$ on velocity gradient. Here, it is observed that the rise in $W e$ turn down the fluid particles motion slowly. Here, upsurge in material relaxation time affords additional struggle to fluid stream which diminishes the velocity gradient. Fig. 4 is devoted to scrutinize the estimation in the velocity profile for several values of $\lambda$. Physically by increasing $\lambda$, the inertial force is overcome by the buoyancy force, and a leading increase is drubbed out for the velocity gradient. Fig. 5 is engrossed to depict the levering of $R b$ on the velocity gradient. Outcome of the figure reveals that the cumulative values of $R b$ deteriorates the velocity gradient. This deteriorating behaviour is caused by the fact that $R b$ is related to the buoyancy force caused by bioconvection, which allows the velocity profile to decay.

Fig. 6 is displayed to envisage the domination of $A$ on the thermal profile. The inclination in $A$ slowdowns the heat transference. Fig. 7 manifests the characteristic of $B i$ on thermal gradient. Here, the increased values of $B i$ increase the heat transference. Physically describes that even more heating is delivered from the surface to the nanoparticles, resulting in an increase in the temperature gradient. Fig. 8 demonstrates the behaviour of $Q_{0}$ on temperature profile. The larger $Q_{0}$ enhances the thermal profile. In fact, Internal heat generation/absorption either improves or dampens heat transport. A incremental increase in $Q_{0}$ increases thermal gradient, showing mechanically that an increase in heat source intensity contributes to a greater thermal diffusion layer, which may increase thermal gradient. Fig. 9 highlights the outcome of the $R d$ on thermal 
profile. The increase in radiation parameter upsurges the thermal distribution. Here, more heat is applied to active liquid due to upshot in radiation phenomenon. As a result, heat transfer escalates Fig. 10 describes the behaviour of the Pr on thermal profile. The inclination in Pr declines the thermal gradient. Lower Pr equate to higher thermal diffusivity, although higher values result in higher diffusivity. As a consequence of this justification, the temperature is reduced. Shear thickening activity is characterised by gradual thinning of the boundary layer. Furthermore, by extension, the Pr is inversely related to thermal diffusivity, resulting in a decrease in the thermal profile.

The graphical results for mass transference for varied values of the $N b$ is expressed in Fig. 11. Here, mass distribution diminishes for inclined $N b$. Concentration gradient decays after more particles are pushed in the reverse way of the solutal distribution to preserve solution homogeneity. Fig. 12 determines the sway of $N t$ over concentration gradient. The escalation in $N t$ declines the mass transfer. Physically, raising in the $N t$ causes an increase in thermophoretic energy, which raises the fluid temperature as well as the nanoparticle concentration due to the migration of nanoparticles from warm to chill regions. Fig. 13 exhibits the feature of $E_{1}$ on concentration distribution. Larger values of $E_{1}$ enhances the Concentration field. As the $E_{1}$ becomes larger, the modified Arrhenius mechanism decays. This eventually inspires the procreative chemical reaction, which causes the concentration of nanoparticles to increase. Figure 14 exemplifies the fluctuation of the concentration field with $\sigma$. The concentration of species in the boundary layer decreases as the value of the $\sigma$ increases. This is because the chemical reaction in this method consumes the chemical, resulting in a reduction in the concentration profile. Variation in mass transference the Sc portrayed in Fig. 15. Mathematically, a dimensionless number relating mass diffusivity with momentum diffusivity yielding a fluid flow is treated as Schmidt number. These two terms are physically called the hydrodynamic thickness layer and mass transport layer. The maximum concentration of nanoparticles corresponds to the smallest $S c$.

Fig. 16 elucidates the depreciation in the motile microorganism distribution for various values of the bioconvection $L b$. With enlarging value of $L b$, a perceptive motile microorganism profile is observed. Fig. 17 demonstrates the impact of the $P e$ on microorganism profile. A rise in $P e$ results in a poorer motile microorganism profile. Increased Pe induces a reduction in the diffusivity of microorganisms and hence a decrease in the motile density of fluid. Fig. 18 displays 
the variation of skin friction coefficient against $M$ and $\lambda$. Here, escalating values of $M$ declines the coefficient of skin friction. Further, $f$ "(0) acts as an increasing function of $\lambda$. Fig. 19 shows the skin friction coefficient variation against $W e$ and $R b$. Here, escalating values of $W e$ improves the coefficient of skin friction. Further, $f^{\prime \prime}(0)$ acts as a deteriorating function of $R b$. The variation of local Nusselt number against $Q_{0}$ and $A$ is demonstrated in figure 20. Here, local Nusselt number acts as a growing function of $Q_{0}$ and declining function of $A$. The change in local Nusselt number against $B i$ and $A$ is established in figure 21. Here, local Nusselt number acts as a growing function of $B i$ and declining function of $A$. The change in local density number of motile microorganisms against $L b$ and $\mathrm{Pe}$ is established in figure 22. Here, density number of motile microorganisms acts as a growing function of $L b$ and declining function of $P e$. .

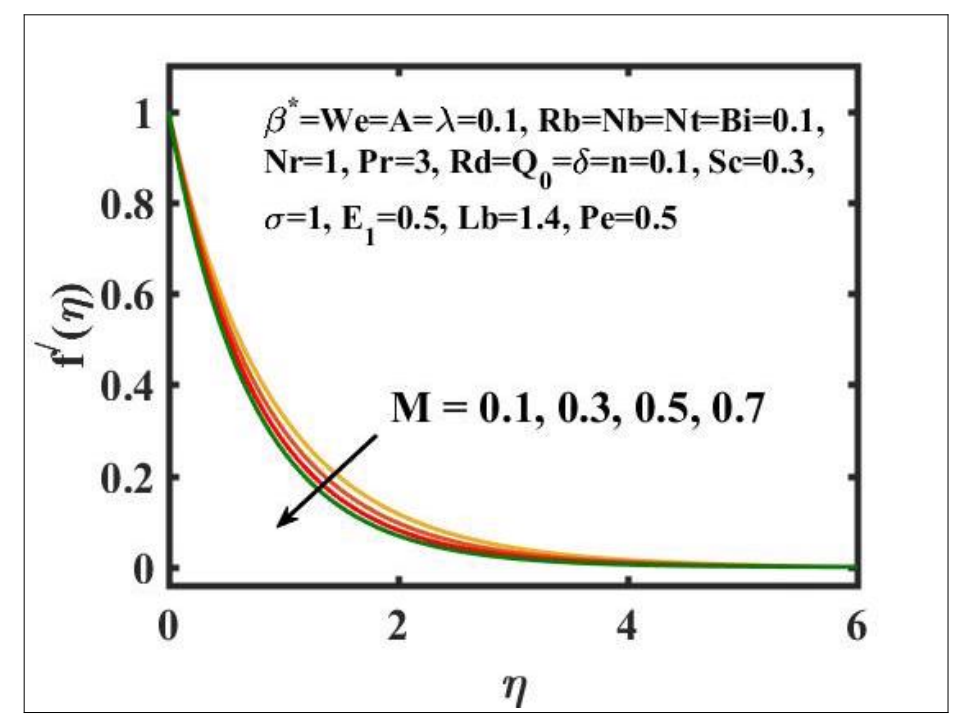

Fig. 2 : Encouragement of $M$ on $f^{\prime}(\eta)$. 


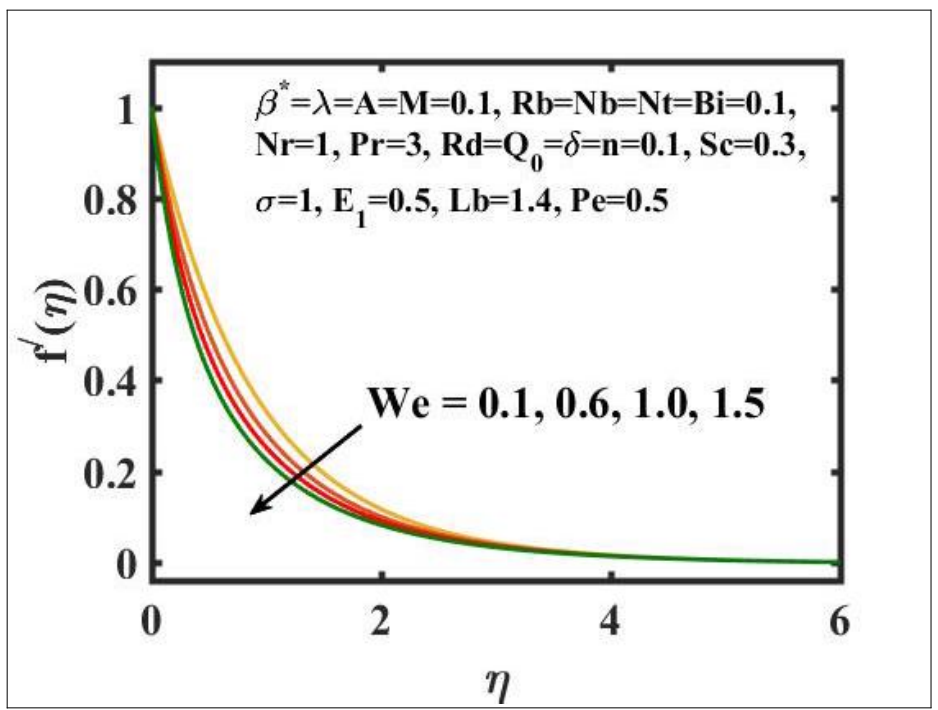

Fig. 3 : Encouragement of $W e$ on $f^{\prime}(\eta)$.

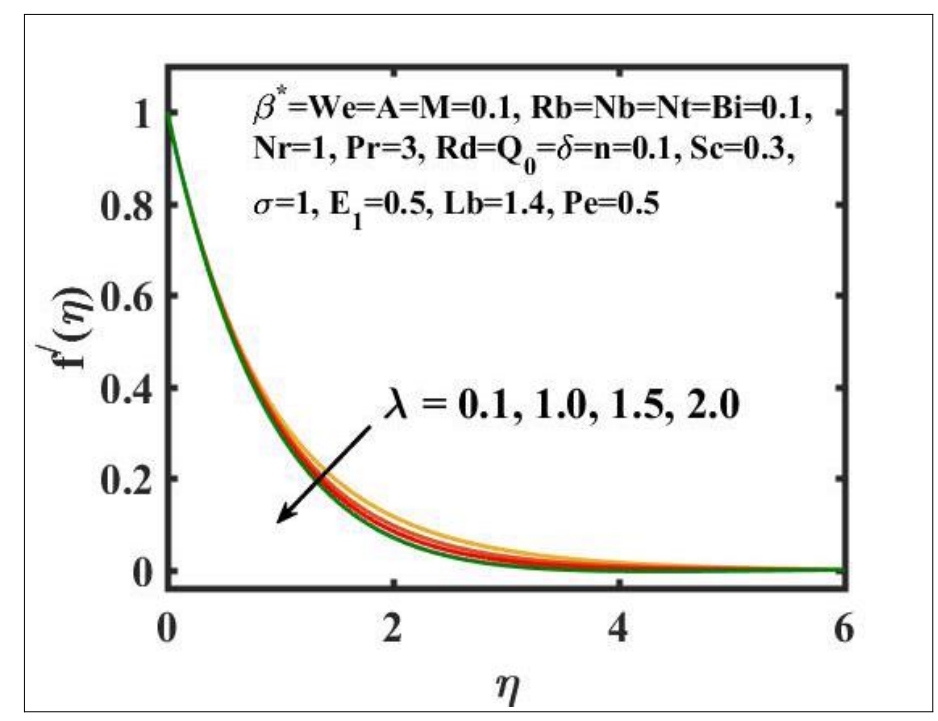

Fig. 4 : Encouragement of $\lambda$ on $f^{\prime}(\eta)$. 


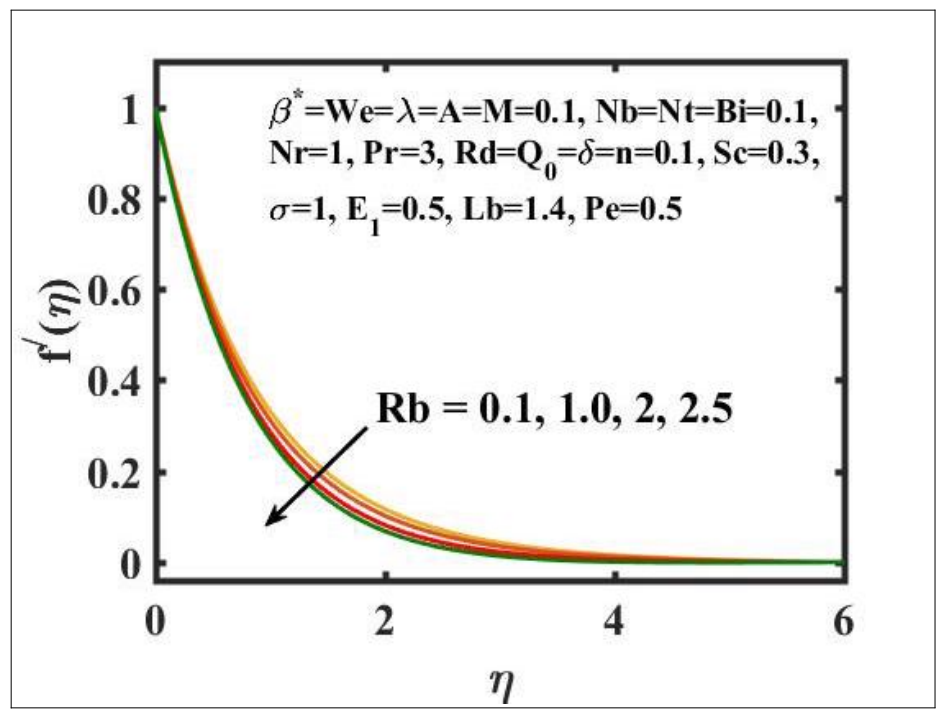

Fig. 5 : Encouragement of $R b$ on $f^{\prime}(\eta)$.

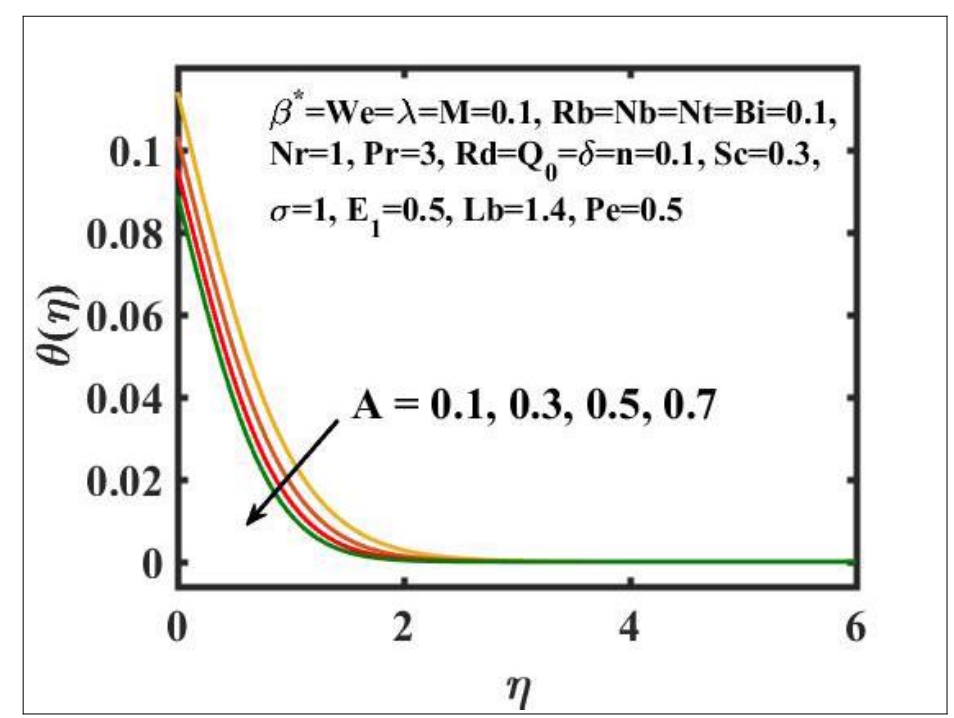

Fig. 6 : Encouragement of $A$ on $\theta(\eta)$. 


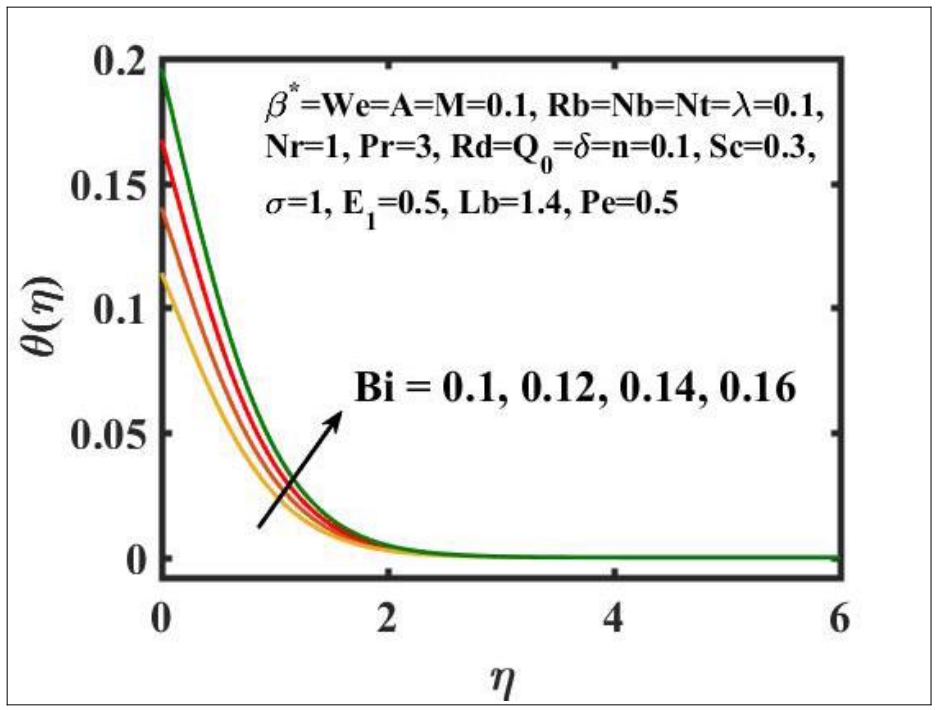

Fig. 7: Encouragement of $B i$ on $\theta(\eta)$.

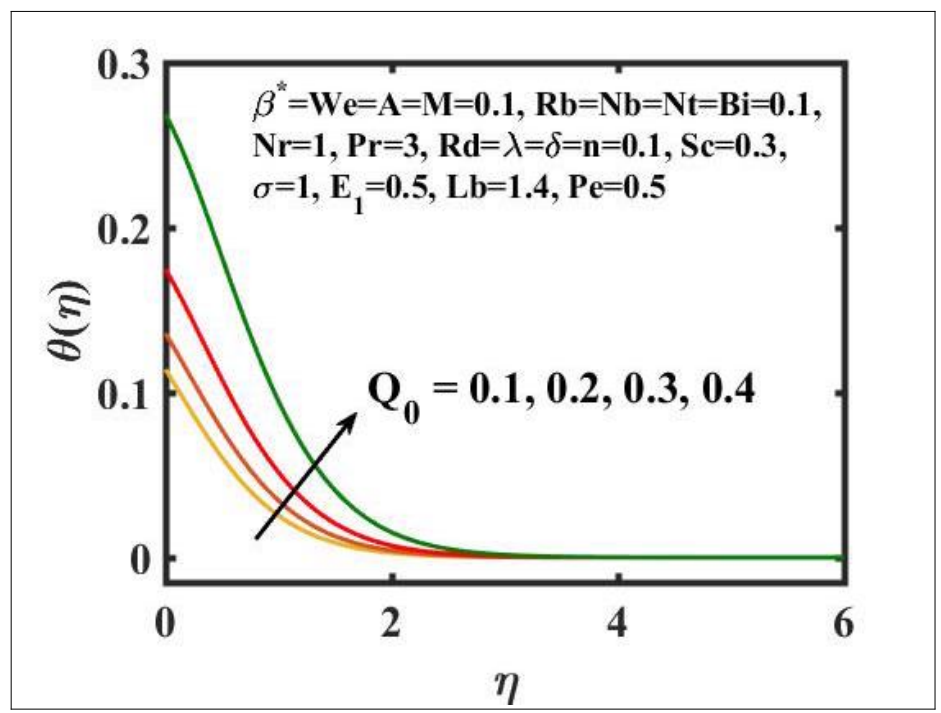

Fig. 8: Encouragement of $Q_{0}$ on $\theta(\eta)$. 


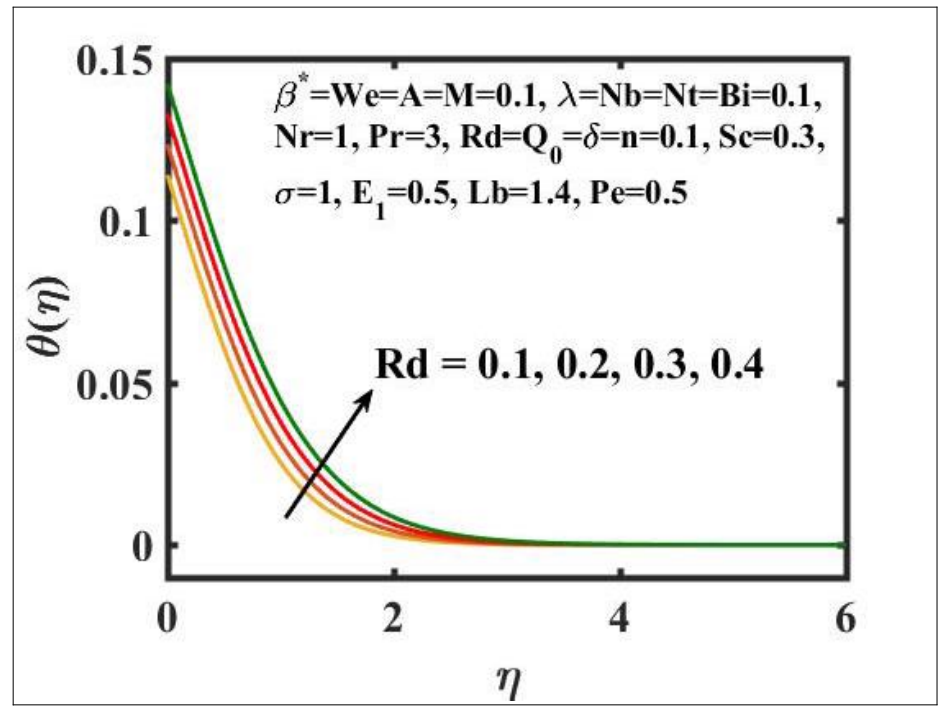

Fig. 9: Encouragement of $R d$ on $\theta(\eta)$.

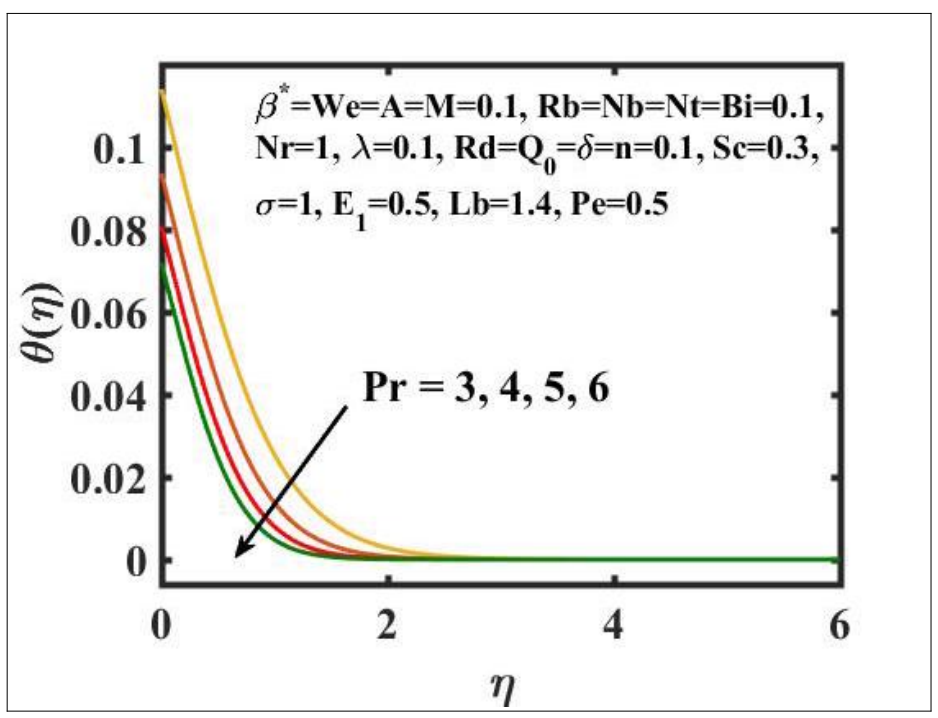

Fig. 10: Encouragement of $\operatorname{Pr}$ on $\theta(\eta)$. 


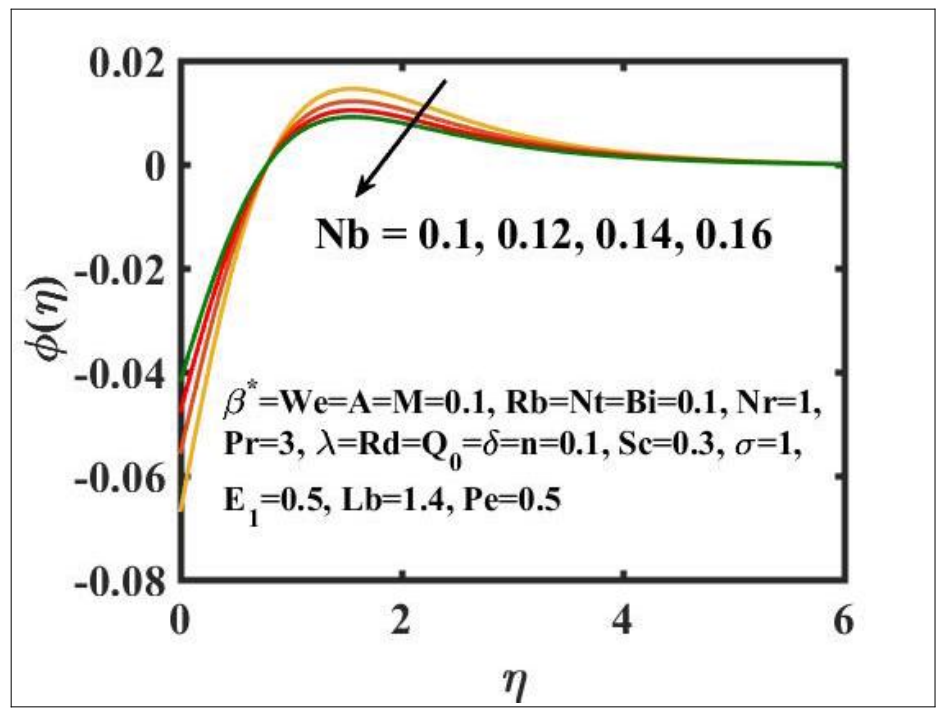

Fig. 11: Encouragement of $N b$ on $\phi(\eta)$.

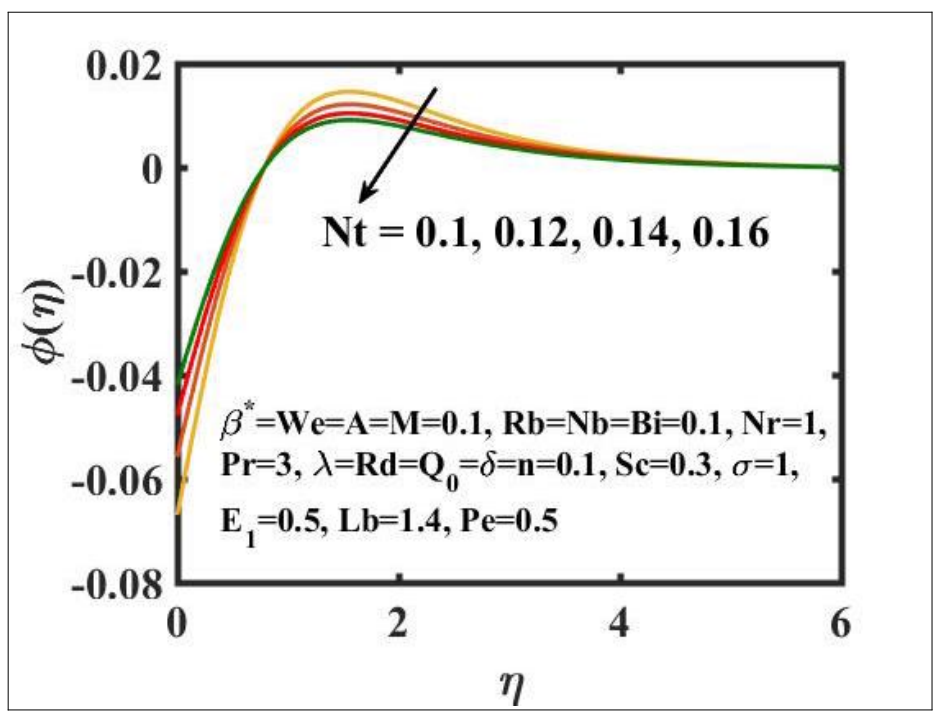

Fig. 12: Encouragement of $N t$ on $\phi(\eta)$. 


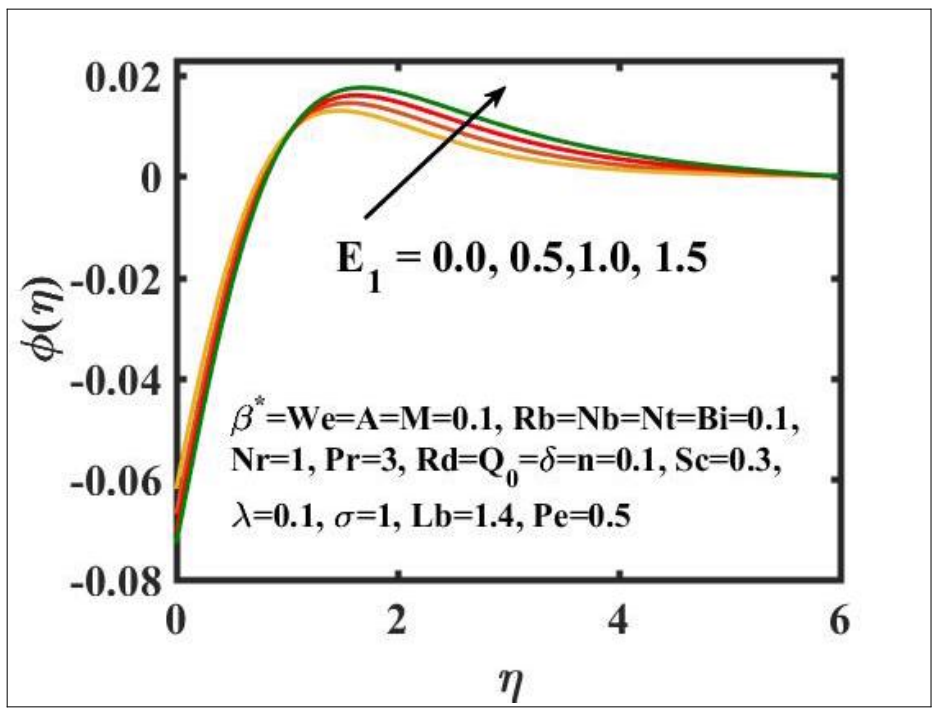

Fig. 13 : Encouragement of $E_{1}$ on $\phi(\eta)$.

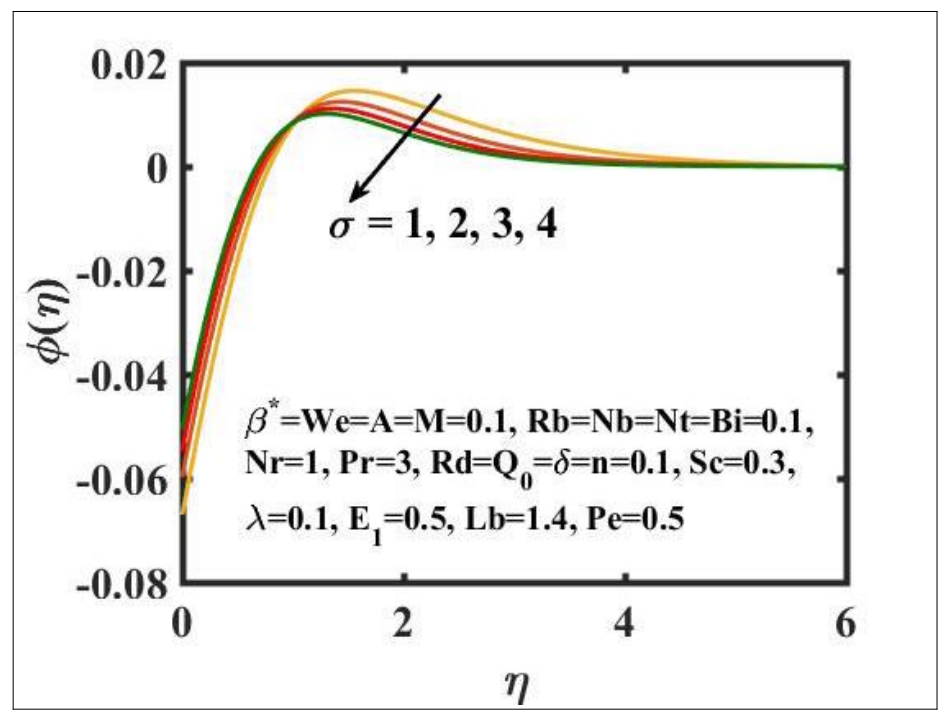

Fig. 14 : Encouragement of $\sigma$ on $\phi(\eta)$. 


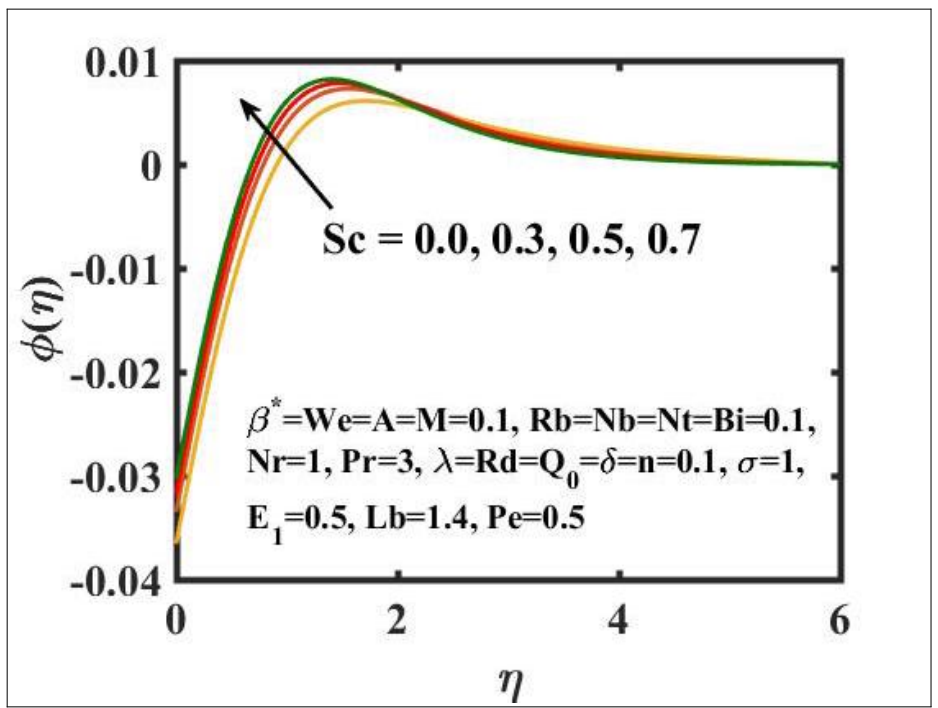

Fig. 15 : Encouragement of $S c$ on $\phi(\eta)$.

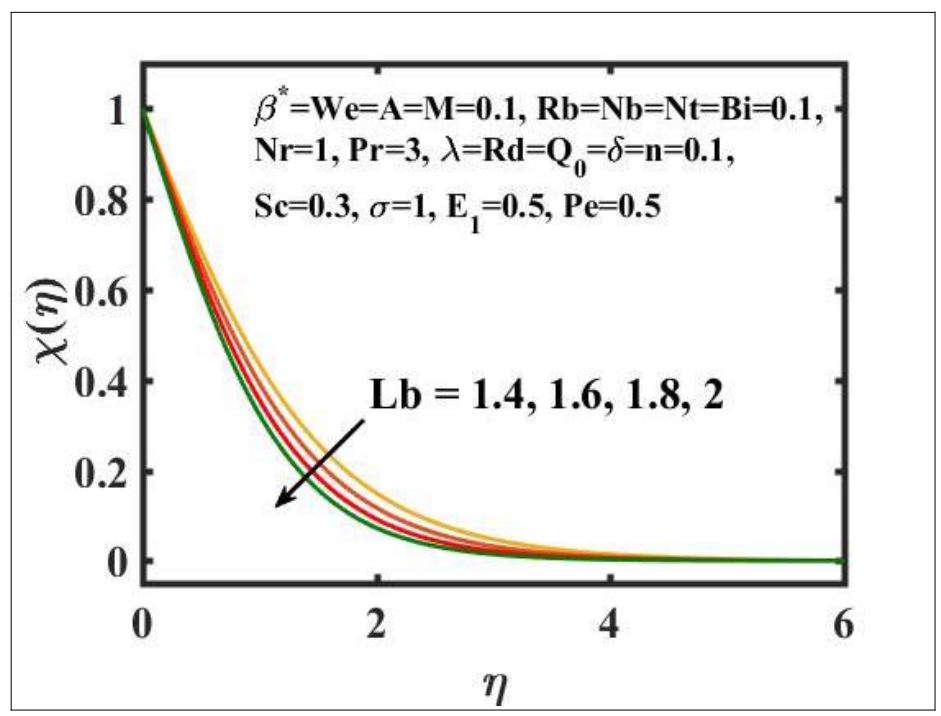

Fig. 16 : Encouragement of $L b$ on $\chi(\eta)$. 


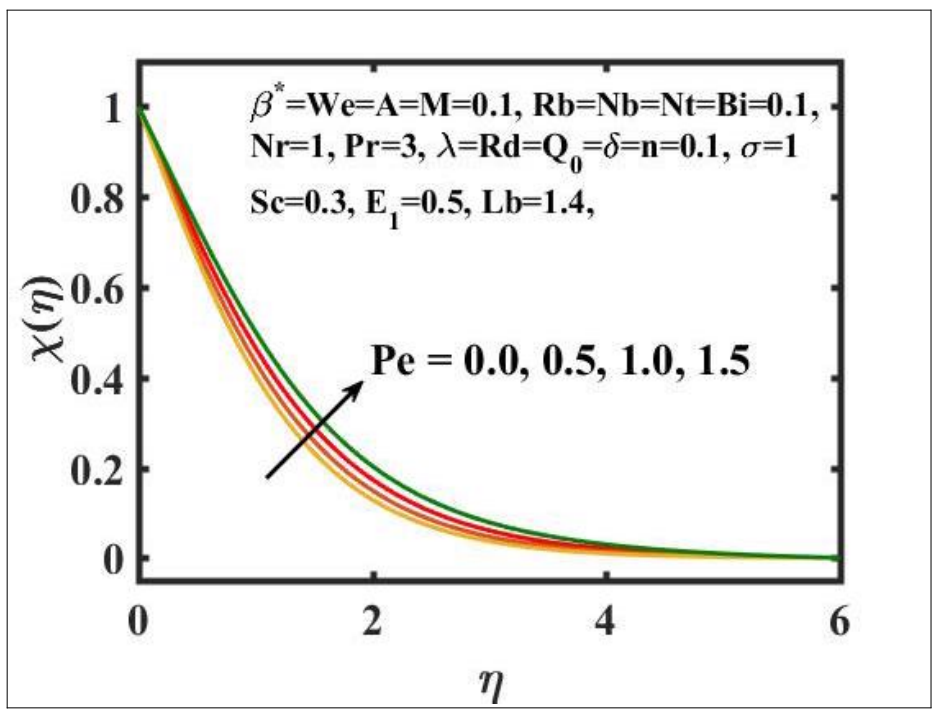

Fig. 17 : Encouragement of $P e$ on $\chi(\eta)$.

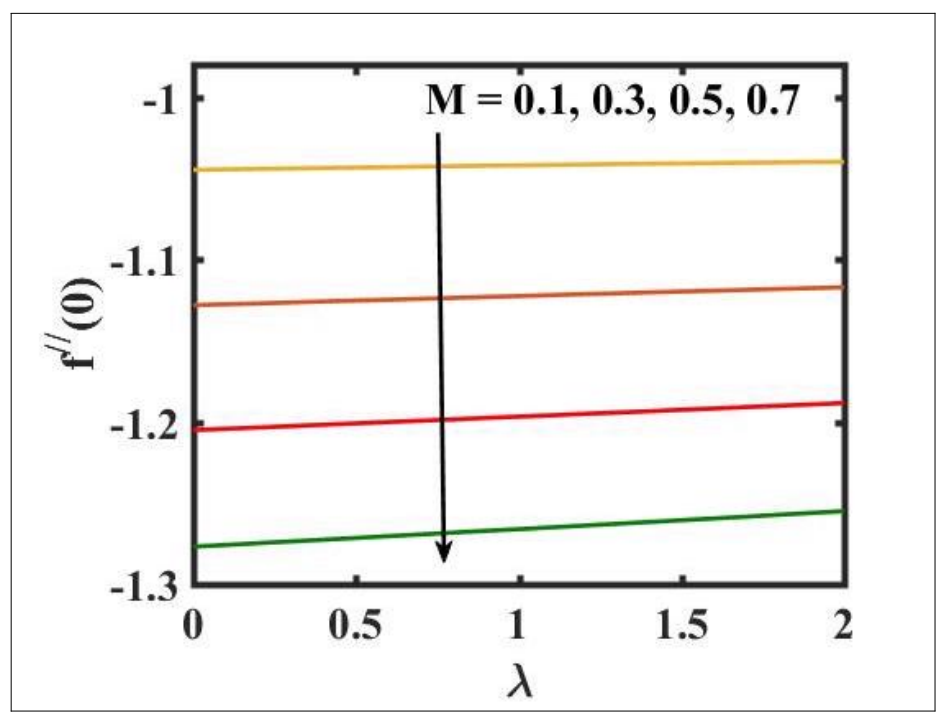

Fig. 18: Encouragement of $M$ and $\lambda$. on skin friction coefficient. 


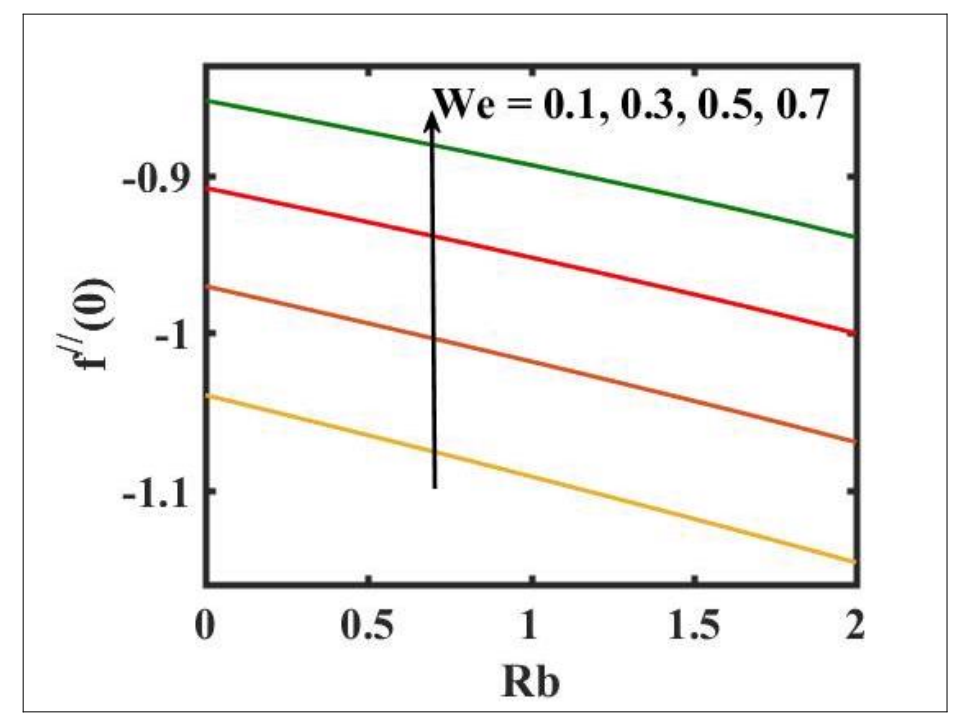

Fig. 19: Encouragement $W e$ and $R b$ on skin friction coefficient

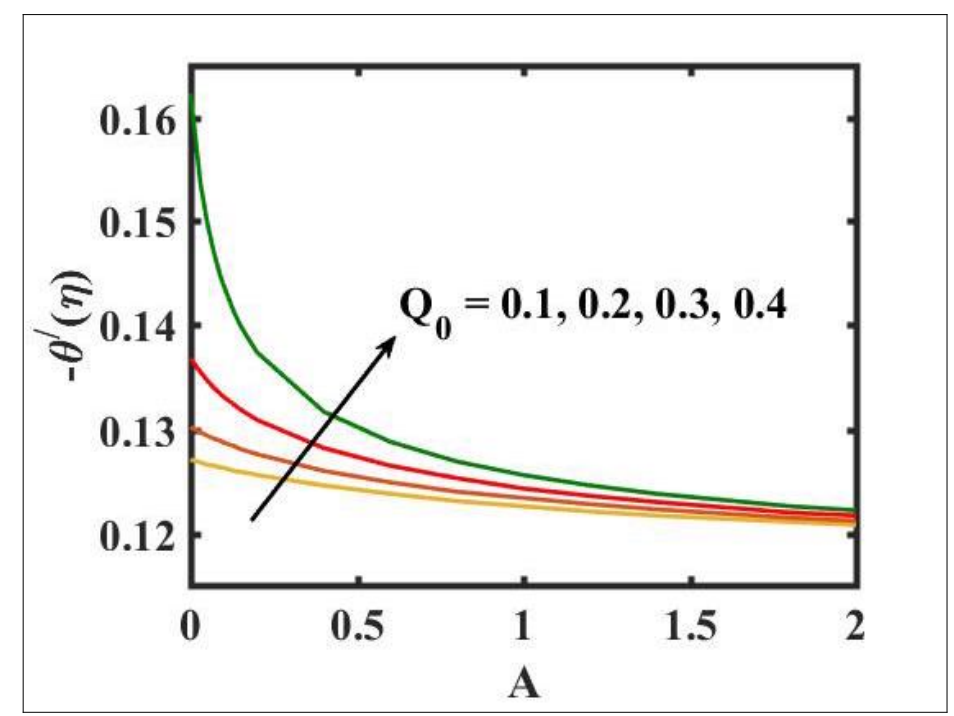

Fig. 20: Encouragement of $Q_{0}$ and $A$ on local Nusselt number. 


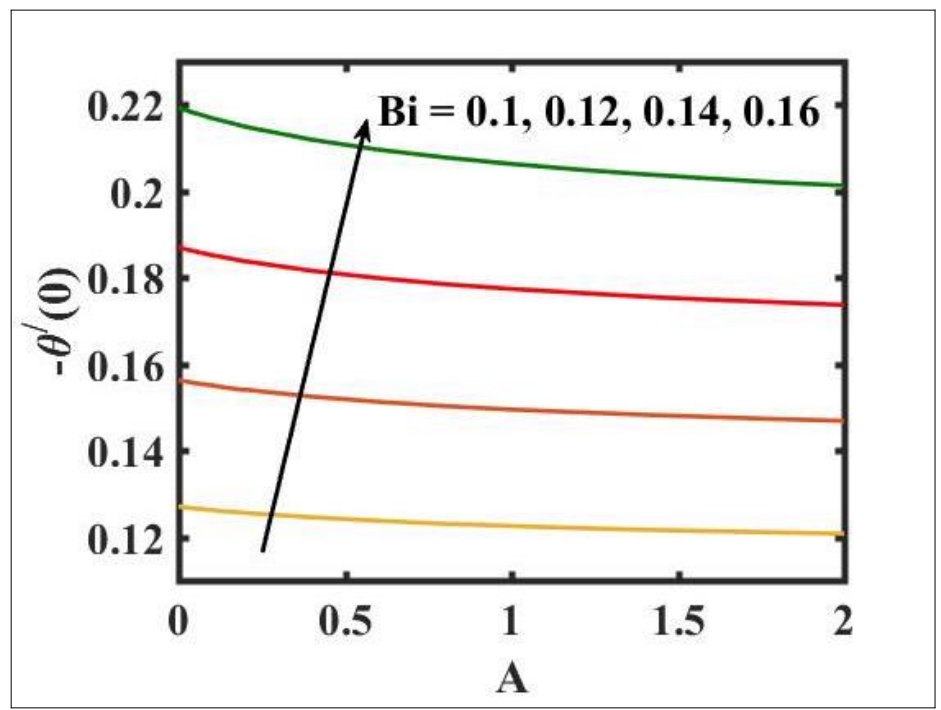

Fig. 21: Encouragement of $B i$ and $A$ local Nusselt number.

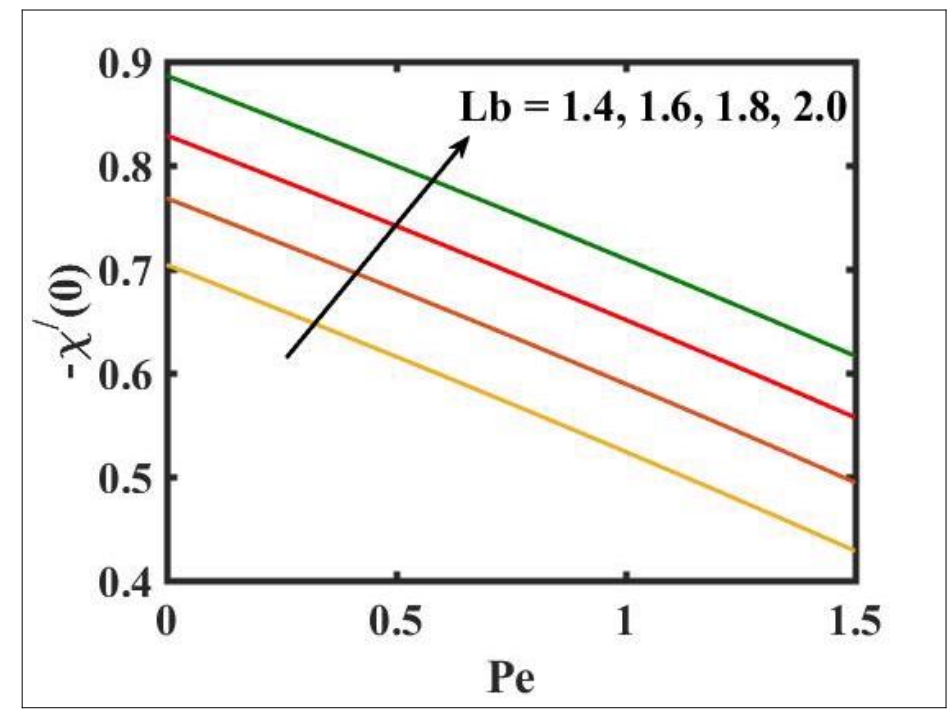

Fig. 22: Encouragement of $L b$ and $P e$ on local density number of motile microorganisms.

\section{Conclusions}

The transient magnetic flow of Cross nanofluid past a stretching sheet with convective boundary condition, thermal radiation effects, binary chemical reactions and heat source/sink is considered in the present model. The effect of Brownian motion and thermophoresis is incorporated into a 
currently developed framework for nanofluid. This section manifests the salient features and the rheological behaviour of various flow physical parameters on velocity, concentration, thermal and motile gyrotactic profile are deliberated graphically. The key conclusions of the current study are:

- The escalation in $M$ and $R b$ slowdowns the velocity and momentum of the fluid.

- The rise in We turn down the fluid particles motion slowly.

- The increased values of $B i$ and $Q_{0}$ intensify the heat transference.

- The increase in radiation parameter upsurges the thermal boundary but, converse trend is seen for escalating Prandtl number.

- The escalation in $N t$ and $N b$ declines the mass transfer.

- An augmentation in the $P e$ and $L b$ deteriorates the microorganism profile.

- The local Nusselt number acts as a growing function of $Q_{0}$ and $B i$ and declining function of $A$.

- The density number of motile microorganisms acts as a growing function of $L b$ and declining function of $P e$.

\section{Data availability Statement}

The data that support the findings of this study are available within the article, the data are made by the authors themselves and do not involve references of others.

\section{References}

[1] M. I. Khan, T. Hayat, M. I. Khan, and A. Alsaedi, 'Activation energy impact in nonlinear radiative stagnation point flow of Cross nanofluid', Int. Commun. Heat Mass Transf., vol. 91, pp. 216-224, Feb. 2018, doi: 10.1016/j.icheatmasstransfer.2017.11.001.

[2] M. Ali, W. A. Khan, M. Irfan, F. Sultan, M. Shahzed, and M. Khan, 'Computational analysis of entropy generation for cross-nanofluid flow', Appl. Nanosci., vol. 10, no. 8, pp. 30453055, Aug. 2020, doi: 10.1007/s13204-019-01038-w.

[3] S. Z. Abbas et al., 'Mathematical modeling and analysis of Cross nanofluid flow subjected to entropy generation', Appl. Nanosci., vol. 10, no. 8, pp. 3149-3160, Aug. 2020, doi: 10.1007/s13204-019-01039-9.

[4] H. Waqas, S. A. Khan, S. U. Khan, M. I. Khan, S. Kadry, and Y.-M. Chu, 'Falkner-Skan 
time-dependent bioconvrction flow of cross nanofluid with nonlinear thermal radiation, activation energy and melting process', Int. Commun. Heat Mass Transf., vol. 120, p. 105028, Jan. 2021, doi: 10.1016/j.icheatmasstransfer.2020.105028.

[5] P.-Y. Xiong et al., 'Dynamics of multiple solutions of Darcy-Forchheimer saturated flow of Cross nanofluid by a vertical thin needle point', Eur. Phys. J. Plus, vol. 136, no. 3, p. 315, Mar. 2021, doi: 10.1140/epjp/s13360-021-01294-2.

[6] T. Hayat, M. Qasim, and S. Mesloub, 'MHD flow and heat transfer over permeable stretching sheet with slip conditions', Int. J. Numer. Methods Fluids, vol. 66, no. 8, pp. 963-975, 2011, doi: https://doi.org/10.1002/fld.2294.

[7] M. Radhika, R. J. P. Gowda, R. Naveenkumar, Siddabasappa, and B. C. Prasannakumara, 'Heat transfer in dusty fluid with suspended hybrid nanoparticles over a melting surface', Heat Transf., vol. n/a, no. n/a, doi: https://doi.org/10.1002/htj.21972.

[8] M. Senapati, K. Swain, and S. Parida, 'Numerical analysis of three-dimensional MHD flow of Casson nanofluid past an exponentially stretching sheet', Karbala Int. J. Mod. Sci., vol. 6, no. 1, Mar. 2020, doi: 10.33640/2405-609X.1462.

[9] V. S. Patil, A. B. Patil, S. Ganesh, P. P. Humane, and N. S. Patil, 'Unsteady MHD flow of a nano powell-eyring fluid near stagnation point past a convectively heated stretching sheet in the existence of chemical reaction with thermal radiation', Mater. Today Proc., Jan. 2021, doi: 10.1016/j.matpr.2020.11.860.

[10] K. Kumaraswamy Naidu, D. Harish Babu, S. Harinath Reddy, and P. V. Satya Narayana, 'Radiation and Partial Slip Effects on Magnetohydrodynamic Jeffrey Nanofluid Containing Gyrotactic Microorganisms Over a Stretching Surface', J. Therm. Sci. Eng. Appl., vol. 13, no. 031011, Sep. 2020, doi: 10.1115/1.4048213.

[11] M. Khan, M. Irfan, W. A. Khan, and A. S. Alshomrani, 'A new modeling for 3D Carreau fluid flow considering nonlinear thermal radiation', Results Phys., vol. 7, pp. 2692-2704, Jan. 2017, doi: 10.1016/j.rinp.2017.07.024.

[12] Hashim, A. Hamid, M. Khan, and U. Khan, 'Thermal radiation effects on Williamson fluid flow due to an expanding/contracting cylinder with nanomaterials: Dual solutions', Phys. Lett. A, vol. 382, no. 30, pp. 1982-1991, Aug. 2018, doi: 10.1016/j.physleta.2018.04.057.

[13] U. Ali, M. Y. Malik, A. A. Alderremy, S. Aly, and K. U. Rehman, 'A generalized findings on thermal radiation and heat generation/absorption in nanofluid flow regime', Phys. Stat. Mech. 
Its Appl., vol. 553, p. 124026, Sep. 2020, doi: 10.1016/j.physa.2019.124026.

[14] M. Gnaneswara Reddy, R. Punith Gowda, R. Naveen Kumar, B. Prasannakumara, and K. Ganesh Kumar, 'Analysis of modified Fourier law and melting heat transfer in a flow involving carbon nanotubes', Proc. Inst. Mech. Eng. Part E J. Process Mech. Eng., p. 09544089211001353 , Mar. 2021, doi: 10.1177/09544089211001353.

[15] M. Ijaz, S. Nadeem, M. Ayub, and S. Mansoor, 'Simulation of magnetic dipole on gyrotactic ferromagnetic fluid flow with nonlinear thermal radiation', J. Therm. Anal. Calorim., vol. 143, no. 3, pp. 2053-2067, Feb. 2021, doi: 10.1007/s10973-020-09856-9.

[16] J. H. Merkin, 'Natural-convection boundary-layer flow on a vertical surface with Newtonian heating', Int. J. Heat Fluid Flow, vol. 15, no. 5, pp. 392-398, Oct. 1994, doi: 10.1016/0142-727X(94)90053-1.

[17] G. Rasool, T. Zhang, A. J. Chamkha, A. Shafiq, I. Tlili, and G. Shahzadi, 'Entropy Generation and Consequences of Binary Chemical Reaction on MHD Darcy-Forchheimer Williamson Nanofluid Flow Over Non-Linearly Stretching Surface', Entropy, vol. 22, no. 1, Art. no. 1, Jan. 2020, doi: 10.3390/e22010018.

[18] J. Wang, W. A. Khan, Z. Asghar, M. Waqas, M. Ali, and M. Irfan, 'Entropy optimized stretching flow based on non-Newtonian radiative nanoliquid under binary chemical reaction', Comput. Methods Programs Biomed., vol. 188, p. 105274, May 2020, doi: 10.1016/j.cmpb.2019.105274.

[19] R. J. Punith Gowda et al., 'Computational modelling of nanofluid flow over a curved stretching sheet using Koo-Kleinstreuer and $\mathrm{Li}(\mathrm{KKL})$ correlation and modified Fourier heat flux model', Chaos Solitons Fractals, vol. 145, p. 110774, Apr. 2021, doi: 10.1016/j.chaos.2021.110774.

[20] N. S. Khan, Z. Shah, M. Shutaywi, P. Kumam, and P. Thounthong, 'A comprehensive study to the assessment of Arrhenius activation energy and binary chemical reaction in swirling flow', Sci. Rep., vol. 10, no. 1, pp. 1-21, 2020.

[21] M. Khan, T. Salahuddin, M. Y. Malik, M. S. Alqarni, and A. M. Alqahtani, 'Numerical modeling and analysis of bioconvection on MHD flow due to an upper paraboloid surface of revolution', Phys. Stat. Mech. Its Appl., vol. 553, p. 124231, Sep. 2020, doi: 10.1016/j.physa.2020.124231.

[22] Y.-M. Chu et al., 'Significance of activation energy, bio-convection and 
magnetohydrodynamic in flow of third grade fluid (non-Newtonian) towards stretched surface: A Buongiorno model analysis', Int. Commun. Heat Mass Transf., vol. 118, p. 104893, Nov. 2020, doi: 10.1016/j.icheatmasstransfer.2020.104893.

[23] K. Al-Khaled, S. U. Khan, and I. Khan, 'Chemically reactive bioconvection flow of tangent hyperbolic nanoliquid with gyrotactic microorganisms and nonlinear thermal radiation', Heliyon, vol. 6, no. 1, p. e03117, Jan. 2020, doi: 10.1016/j.heliyon.2019.e03117.

[24] P. G. R. Jayadevamurthy, N. kumar Rangaswamy, B. C. Prasannakumara, and K. S. Nisar, 'Emphasis on unsteady dynamics of bioconvective hybrid nanofluid flow over an upwarddownward moving rotating disk', Numer. Methods Partial Differ. Equ., vol. n/a, no. n/a, doi: https://doi.org/10.1002/num.22680.

[25] A. A. M. Arafa, Z. Z. Rashed, and S. E. Ahmed, 'Radiative MHD bioconvective nanofluid flow due to gyrotactic microorganisms using Atangana-Baleanu Caputo fractional derivative', Phys. Scr., vol. 96, no. 5, p. 055211, Mar. 2021, doi: 10.1088/1402-4896/abe82d. 


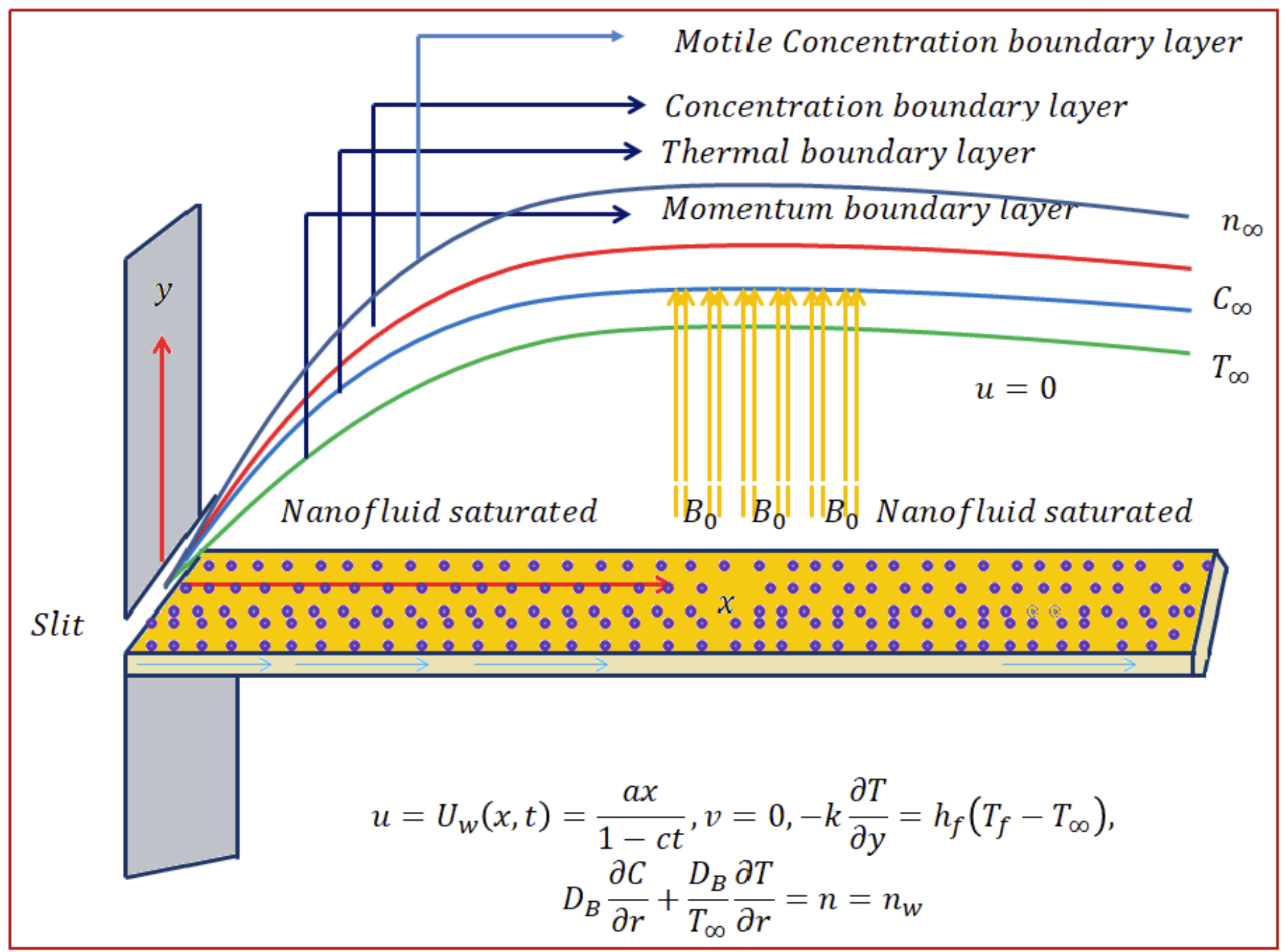

Figure 1

Schematic sketch of the physical model. 


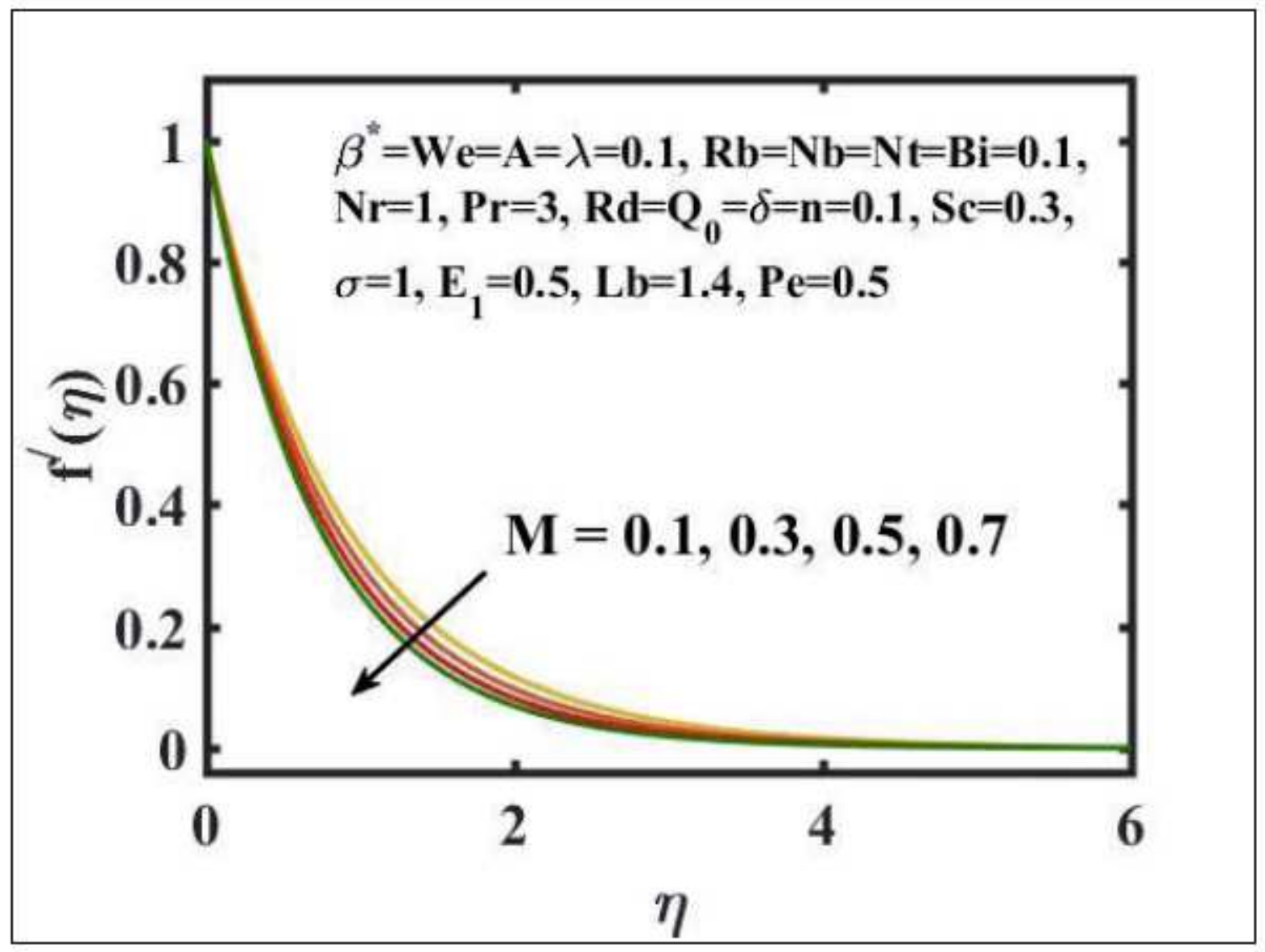

Figure 2

Encouragement of $M$ on $f n$. 


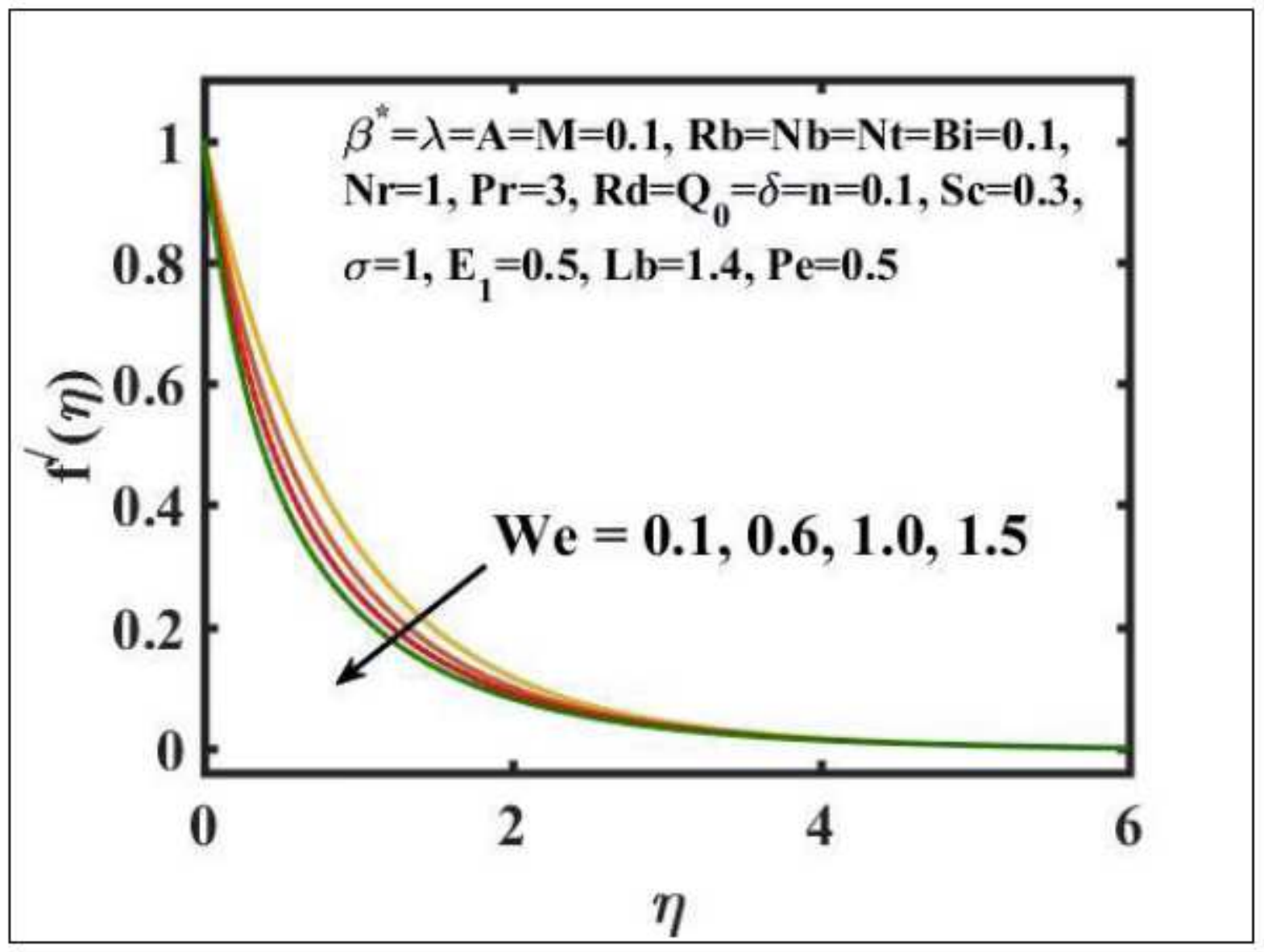

Figure 3

Encouragement of We on $\mathrm{fn}$. 


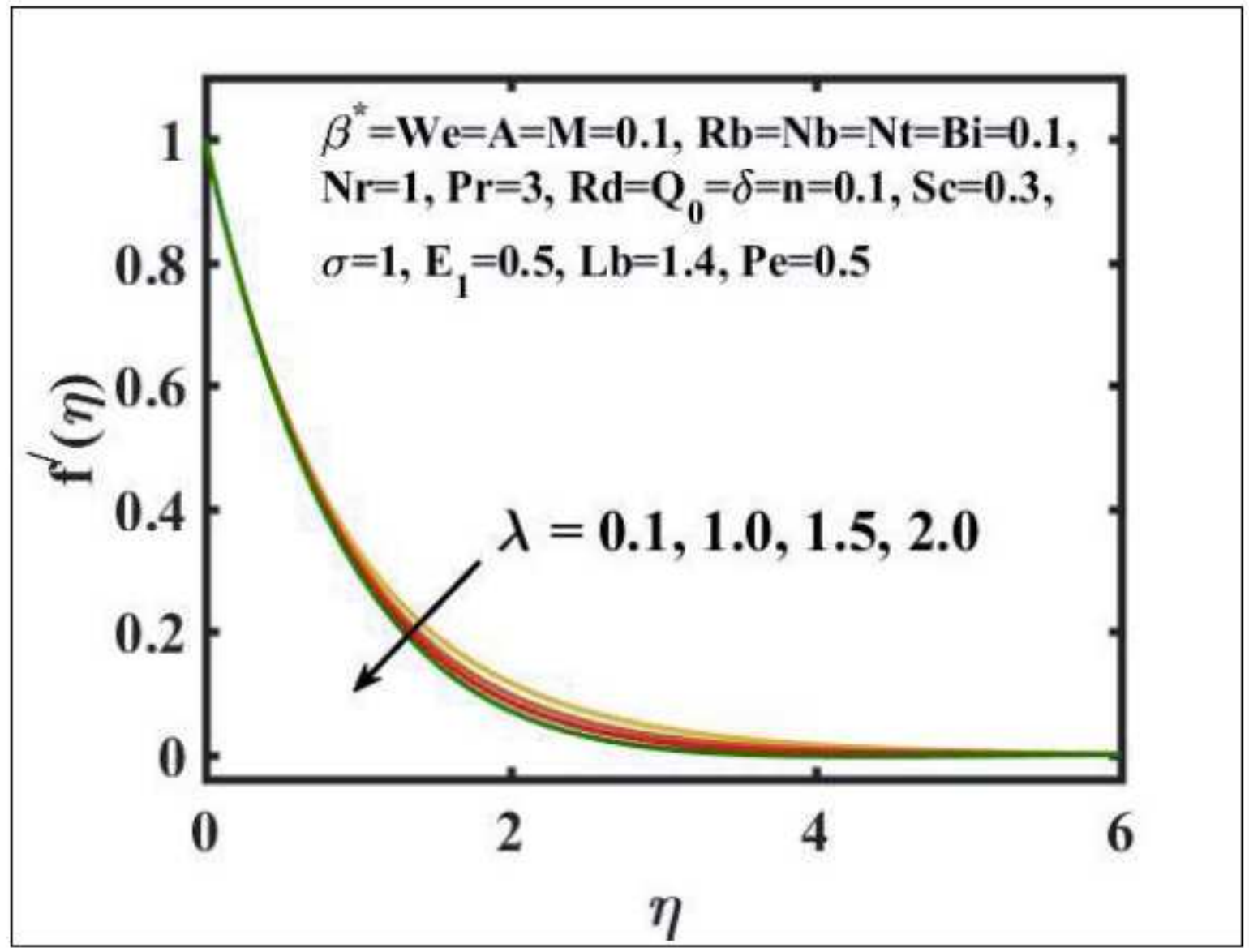

Figure 4

Encouragement of $\lambda$ on $f^{\prime} n$. 


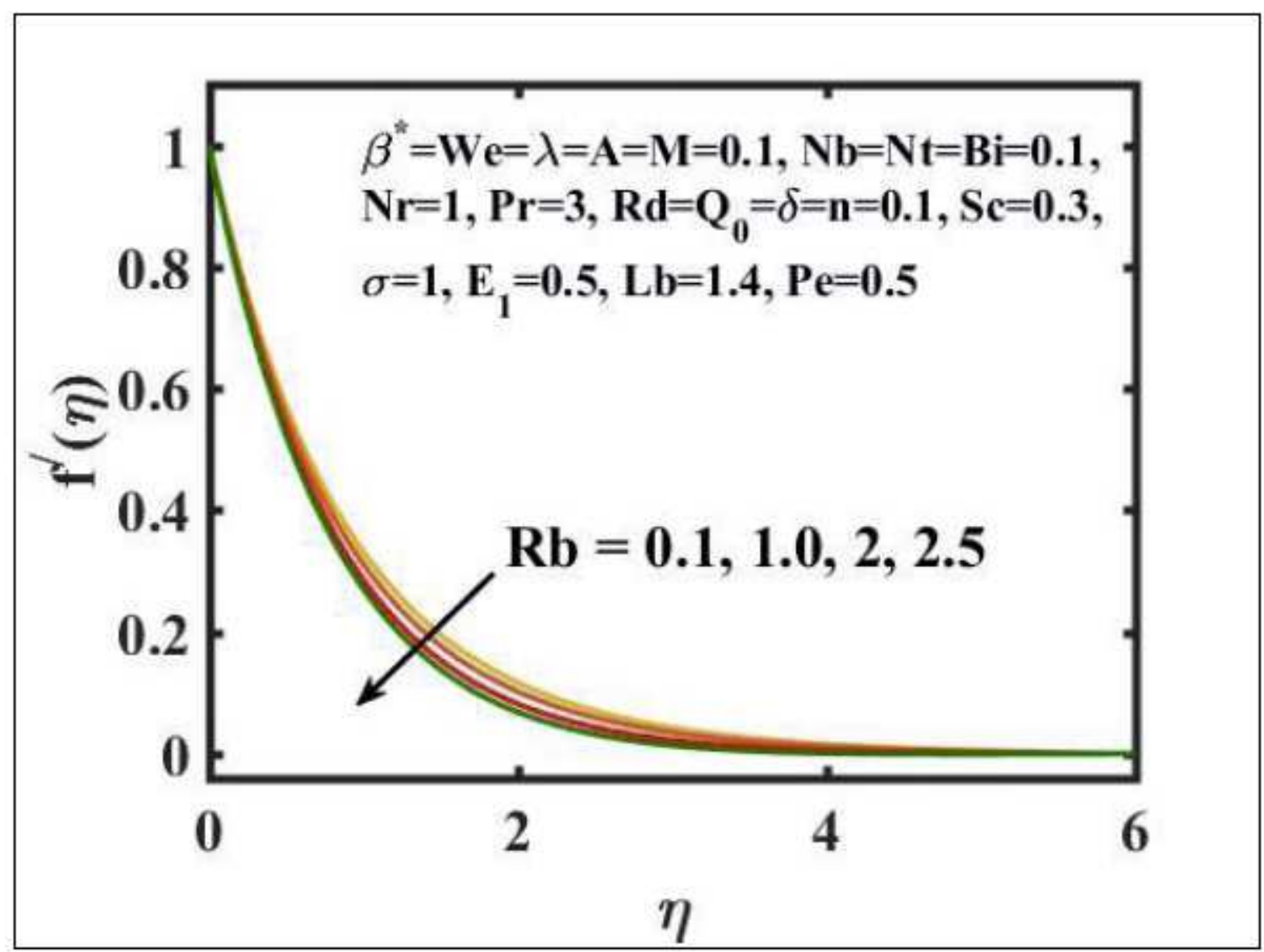

Figure 5

Encouragement of Rb on f'n. 


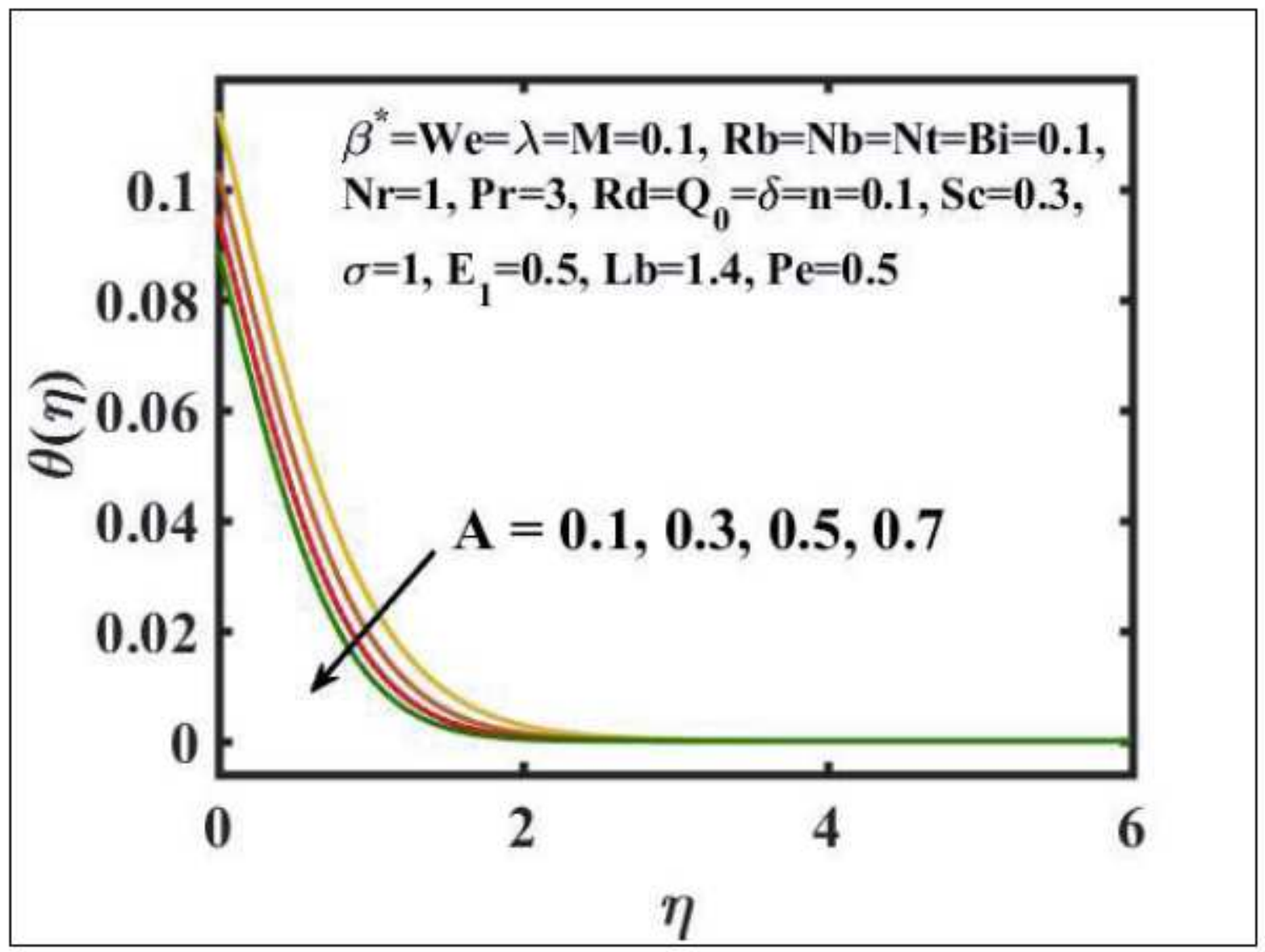

Figure 6

Encouragement of $A$ on $\varnothing(n)$ 


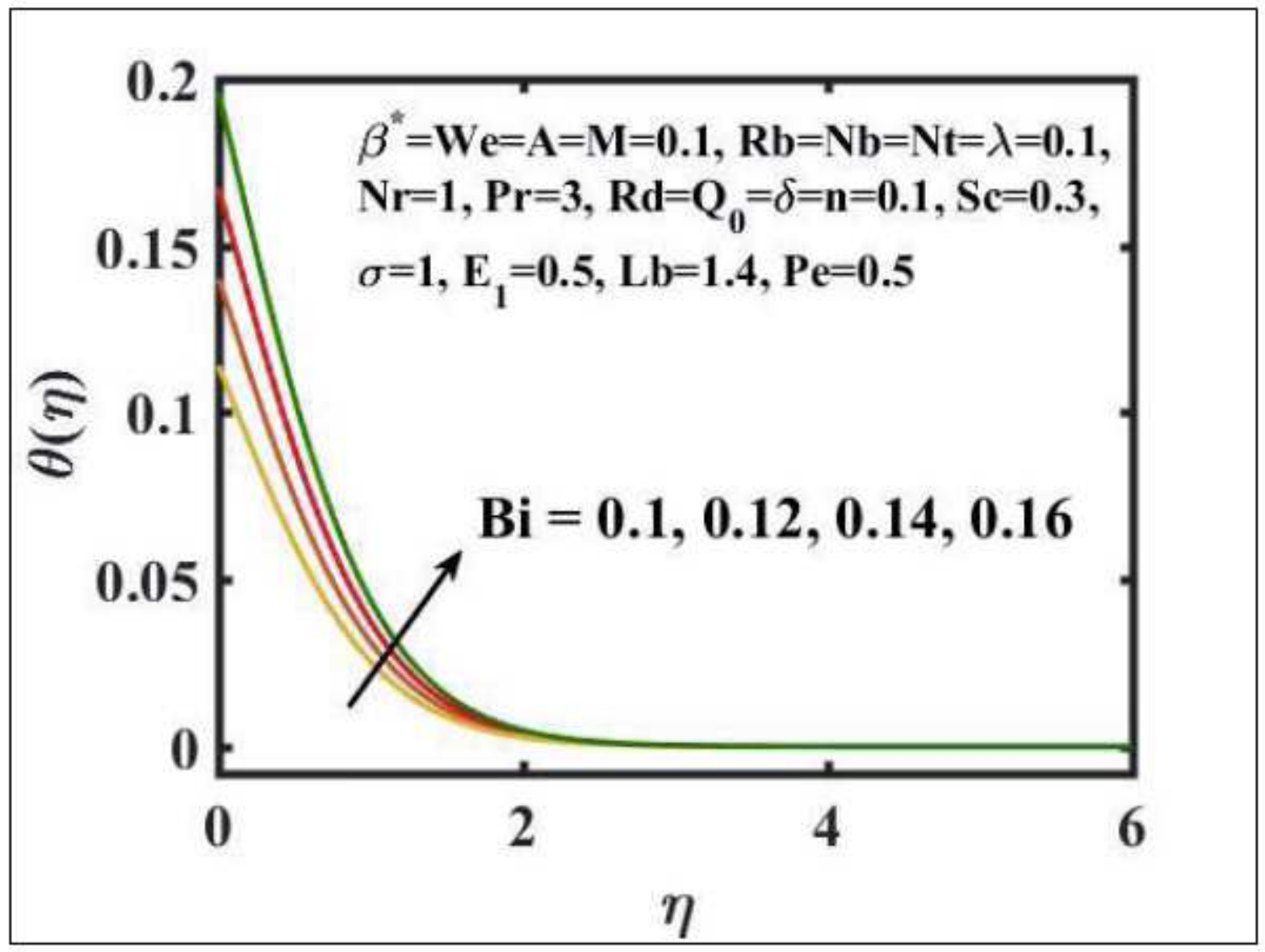

Figure 7

Encouragement of $\mathrm{Bi}$ on $\varnothing(\mathrm{n})$ 


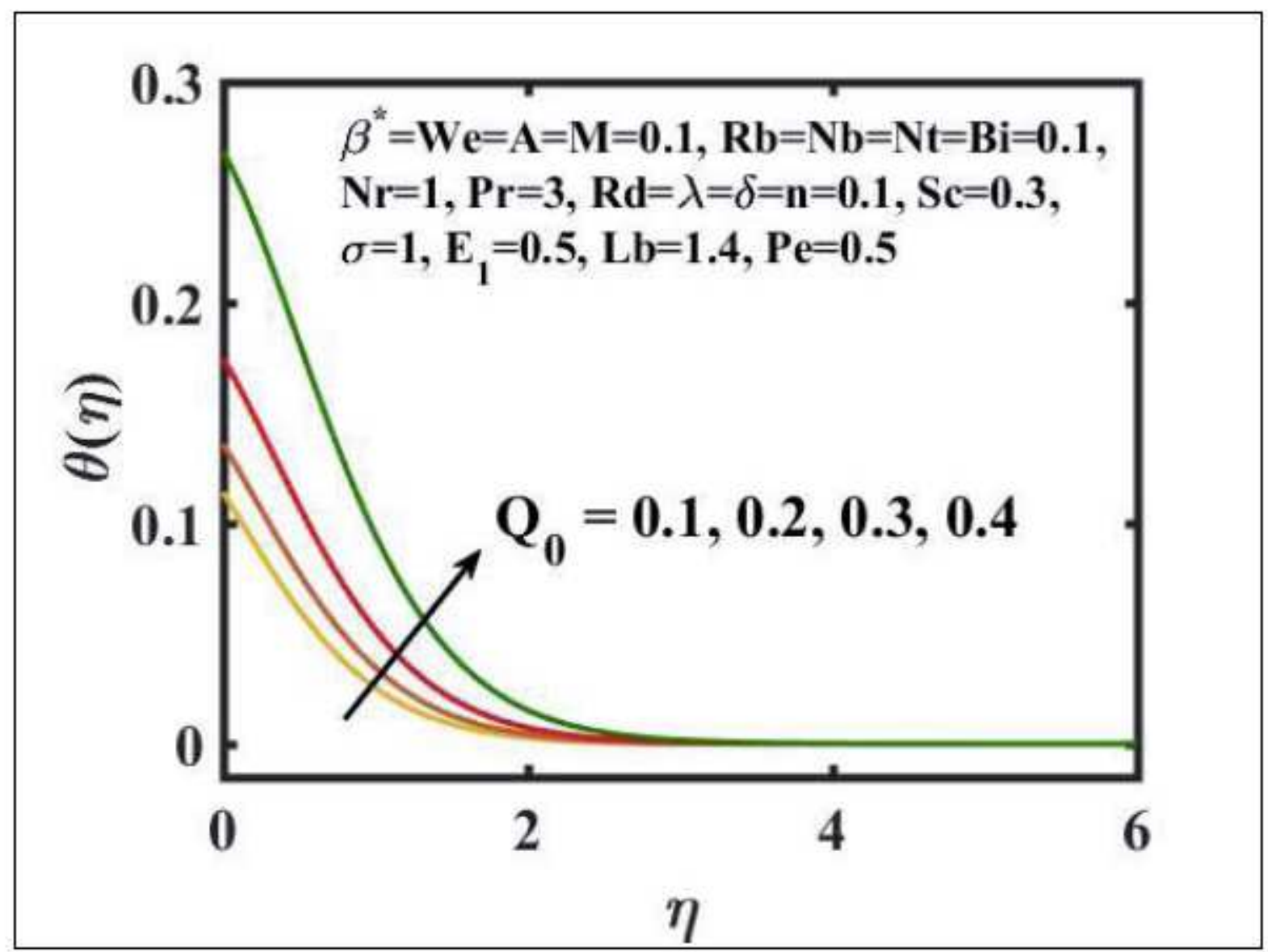

Figure 8

Encouragement of Q0 on $\varnothing(n)$ 


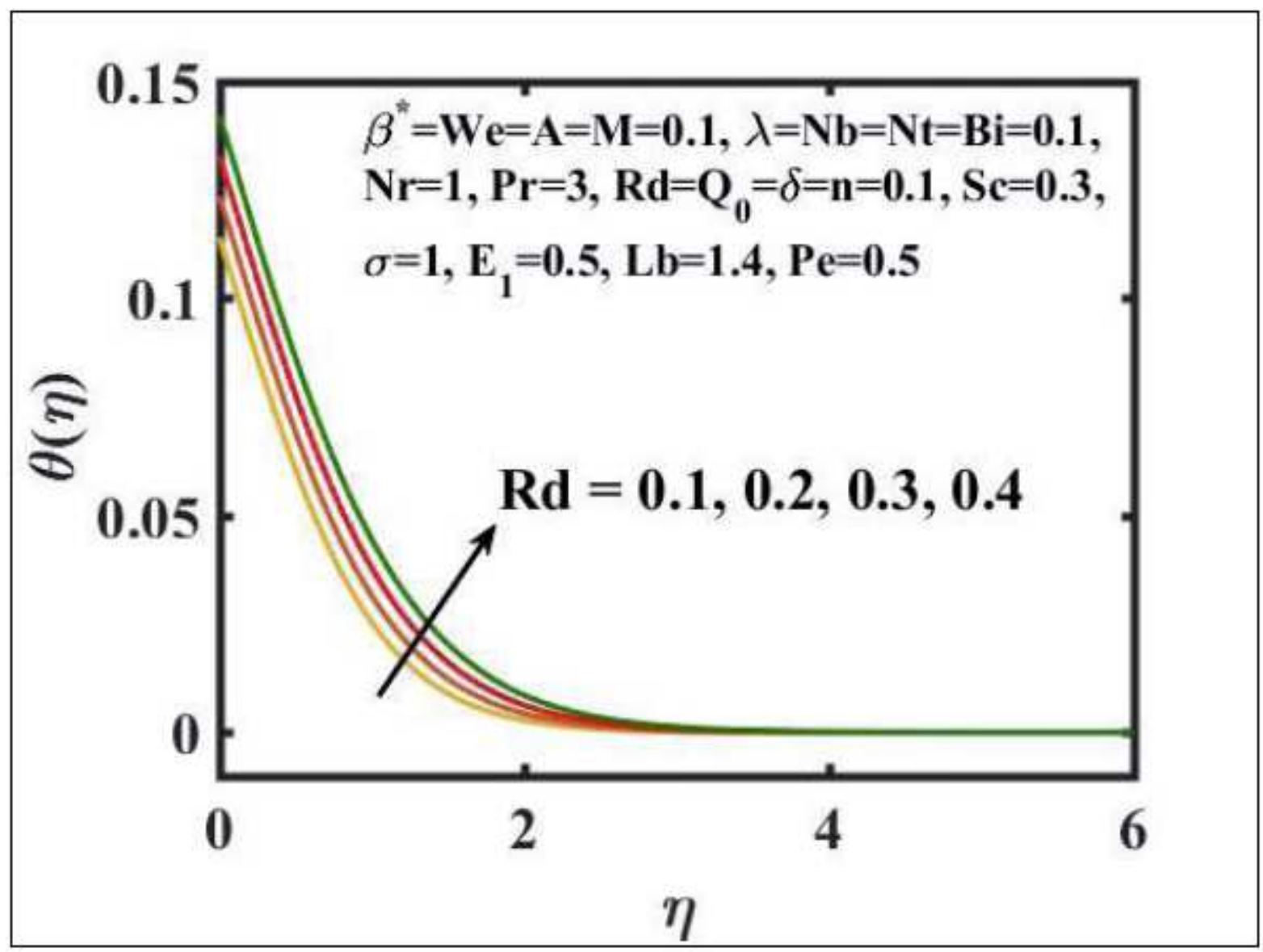

Figure 9

Encouragement of Rd on $\varnothing(n)$ 


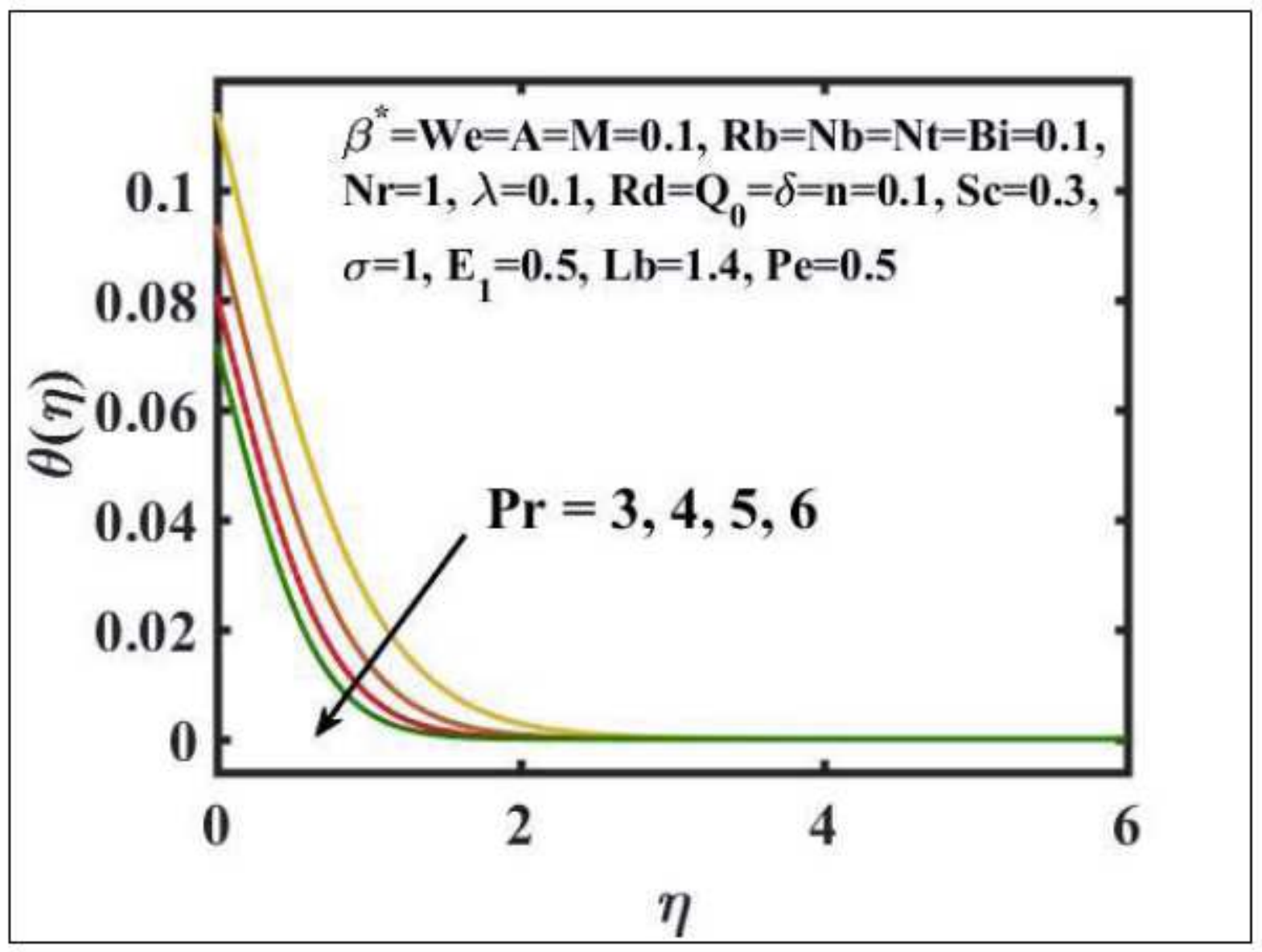

Figure 10

Encouragement of $\operatorname{Pr}$ on $\varnothing(n)$ 


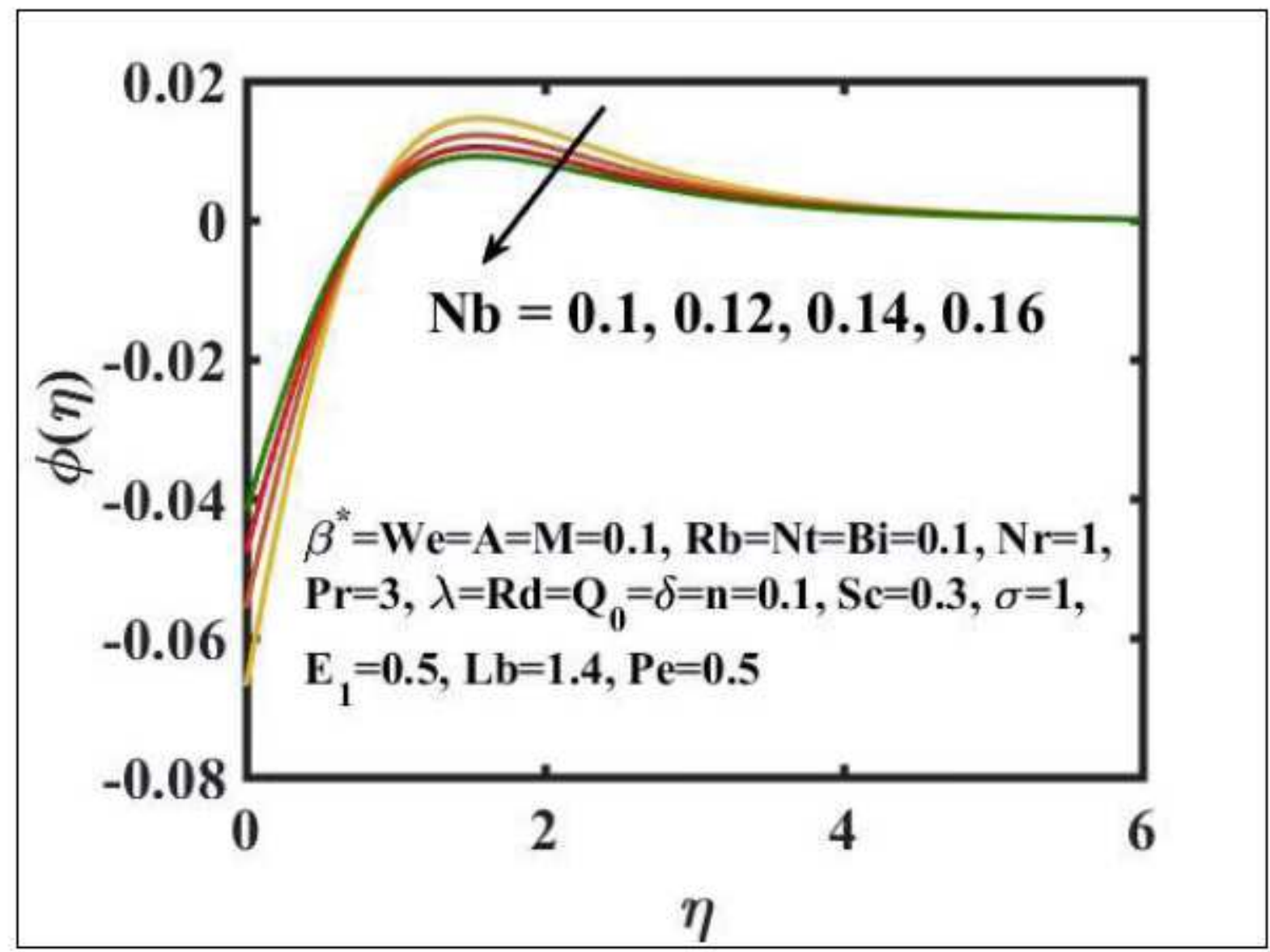

Figure 11

Encouragement of $\mathrm{Nb}$ on $\emptyset(\mathrm{n})$ 


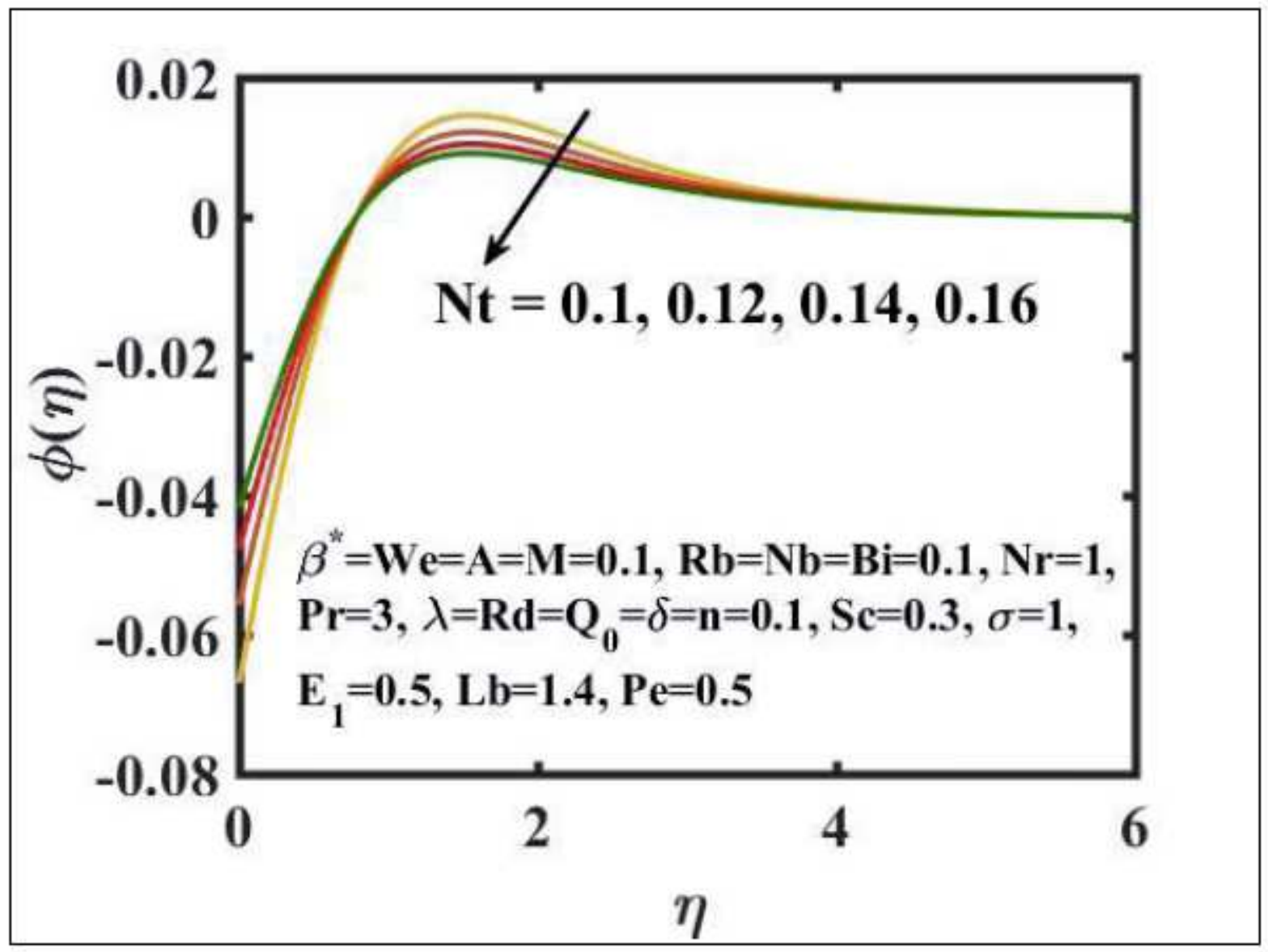

Figure 12

Encouragement of Nt on $\varnothing(n)$ 


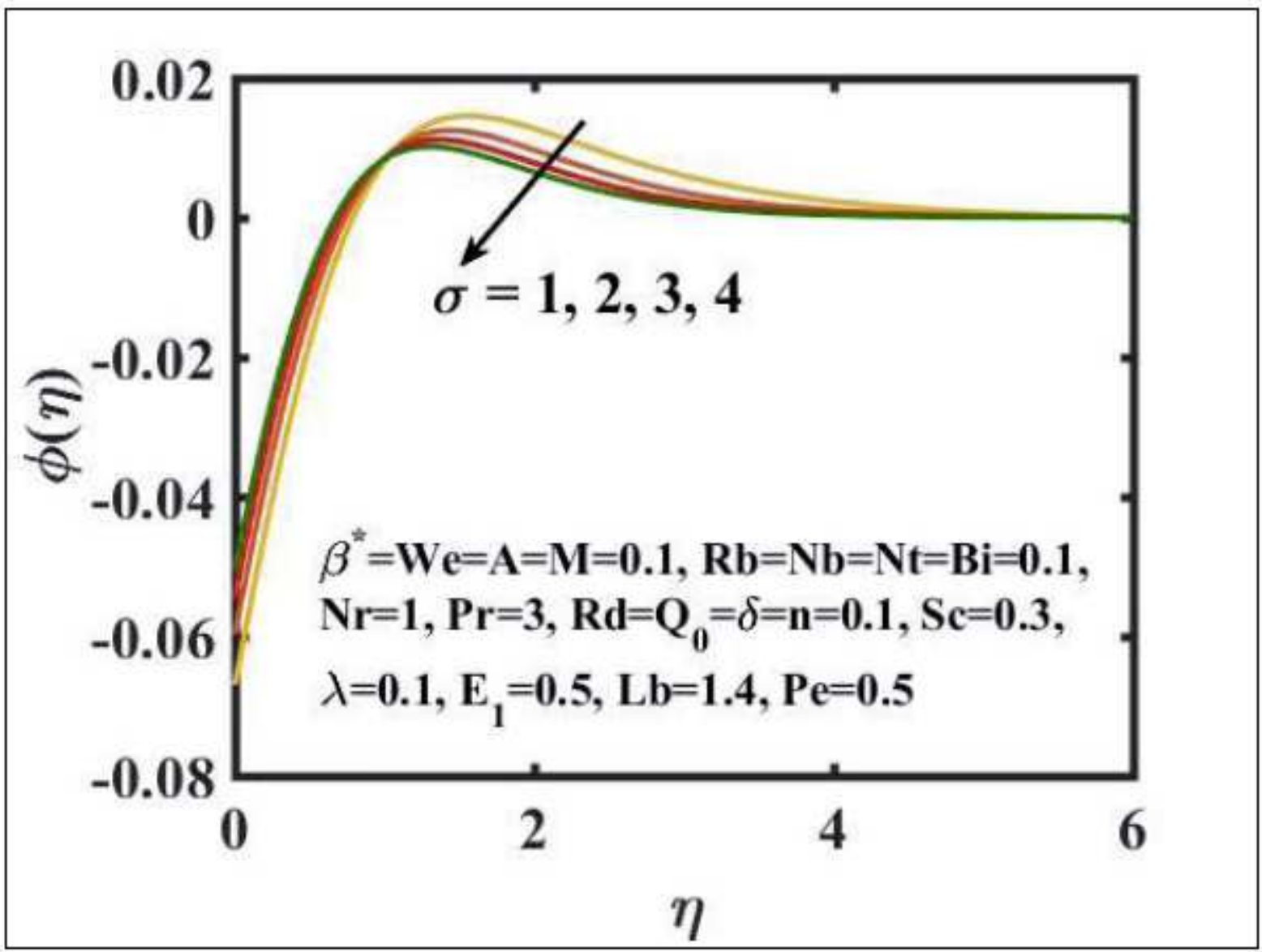

Figure 13

Encouragement of E1 on $\varnothing(n)$ 


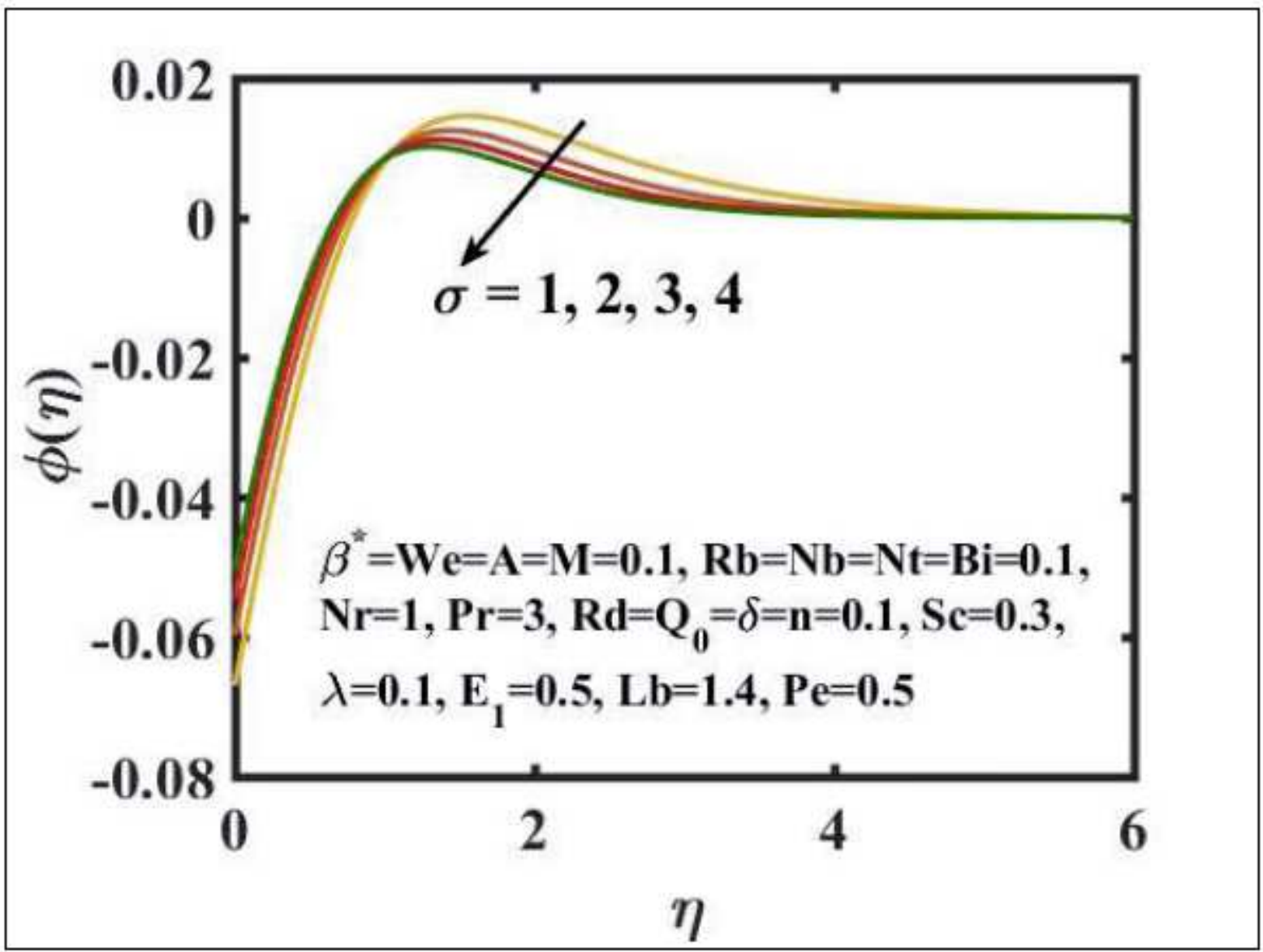

Figure 14

Encouragement of $\sigma$ on $\varnothing(n)$ 


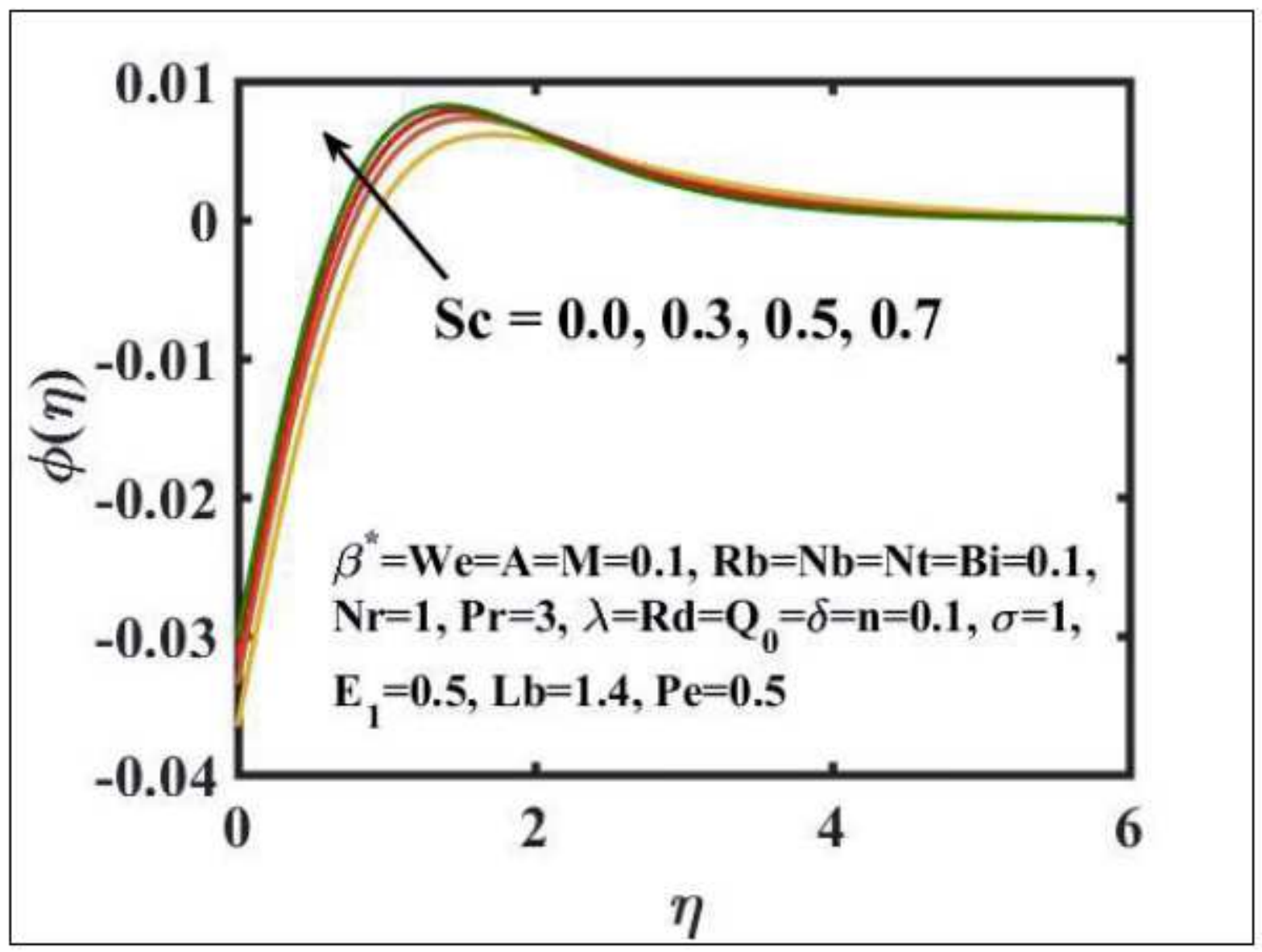

Figure 15

Encouragement of $S c$ on $\varnothing(n)$ 


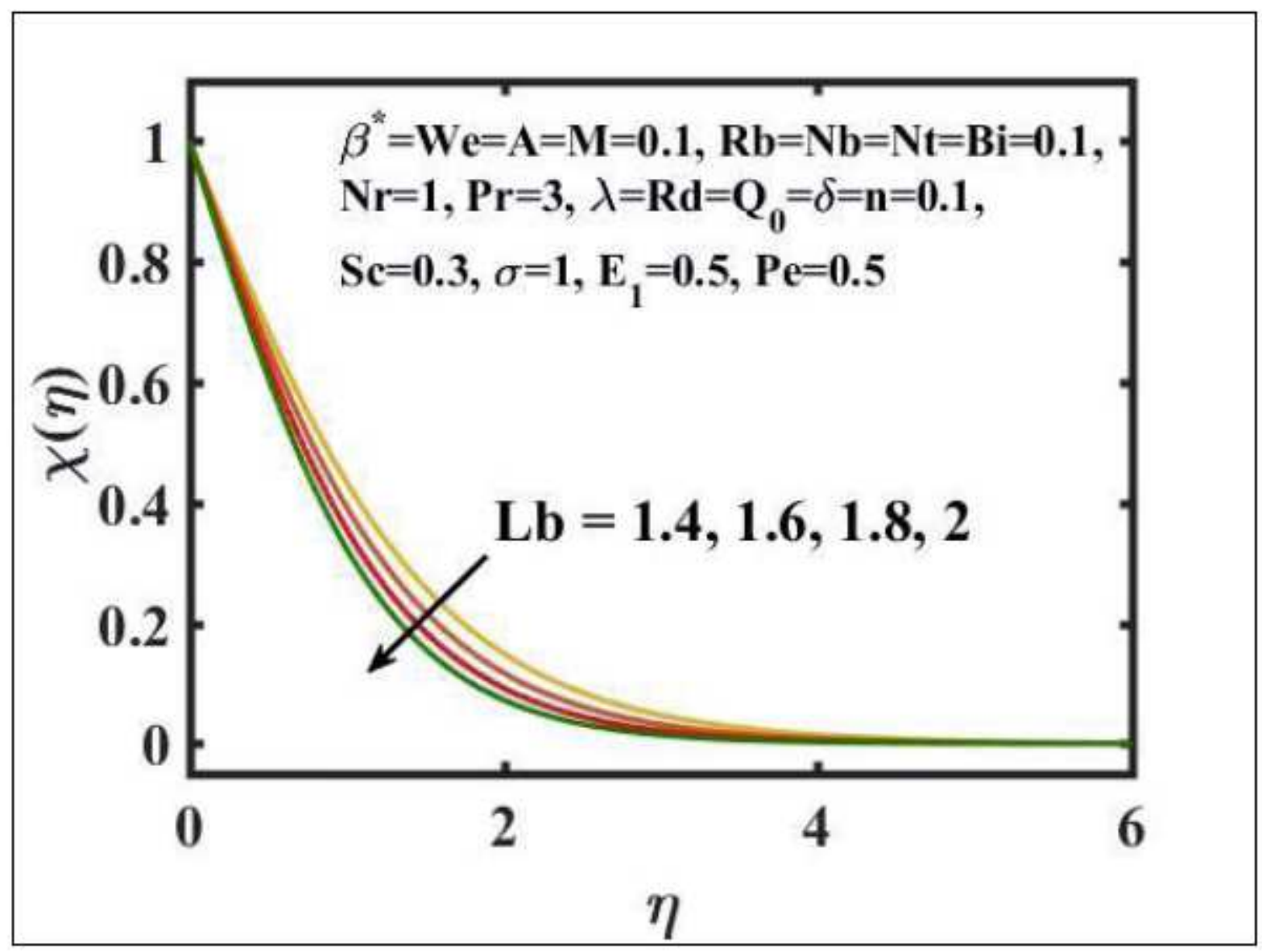

Figure 16

Encouragement of Lb on X (n). 


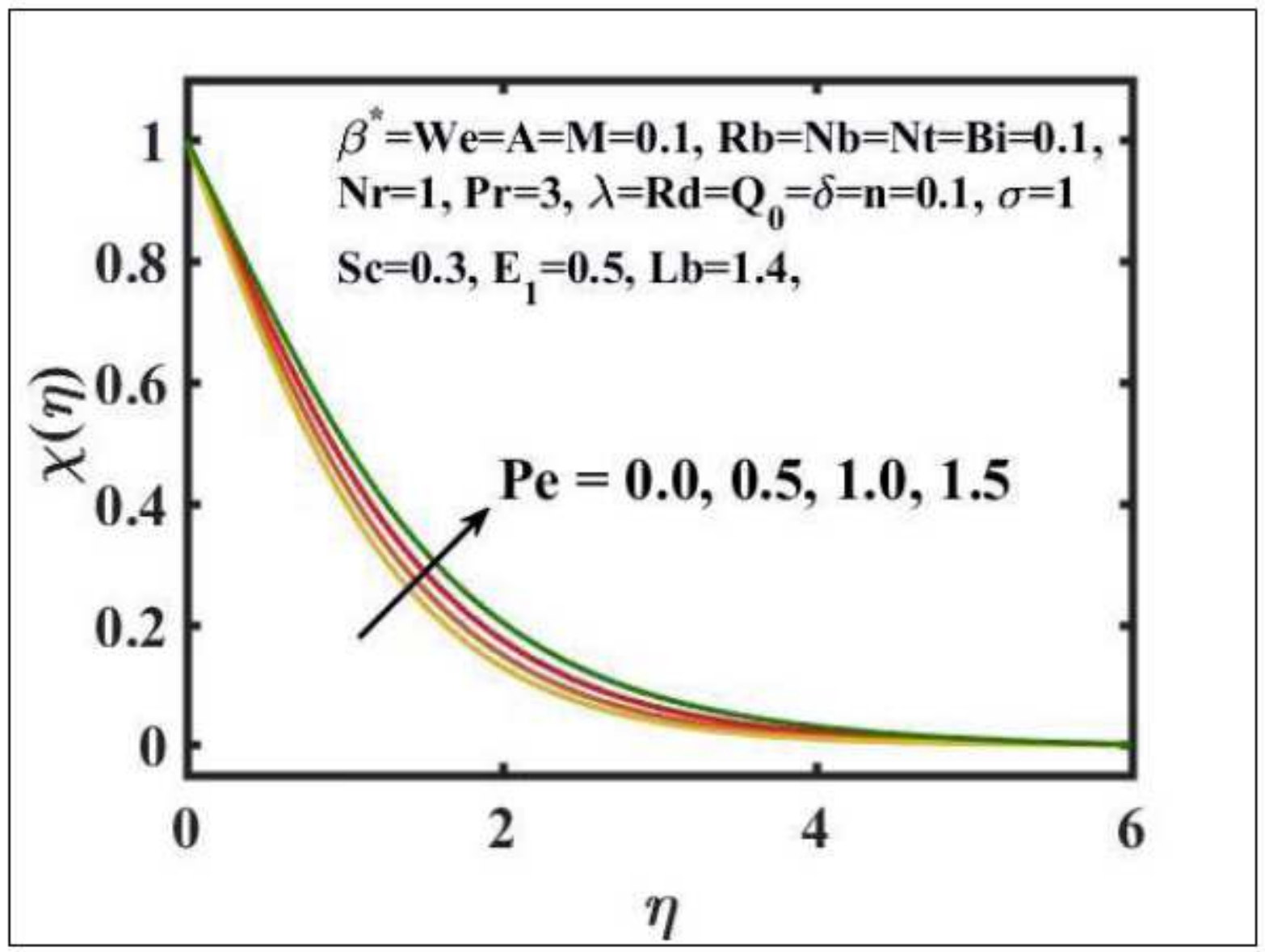

Figure 17

Encouragement of Pe on X(n) 


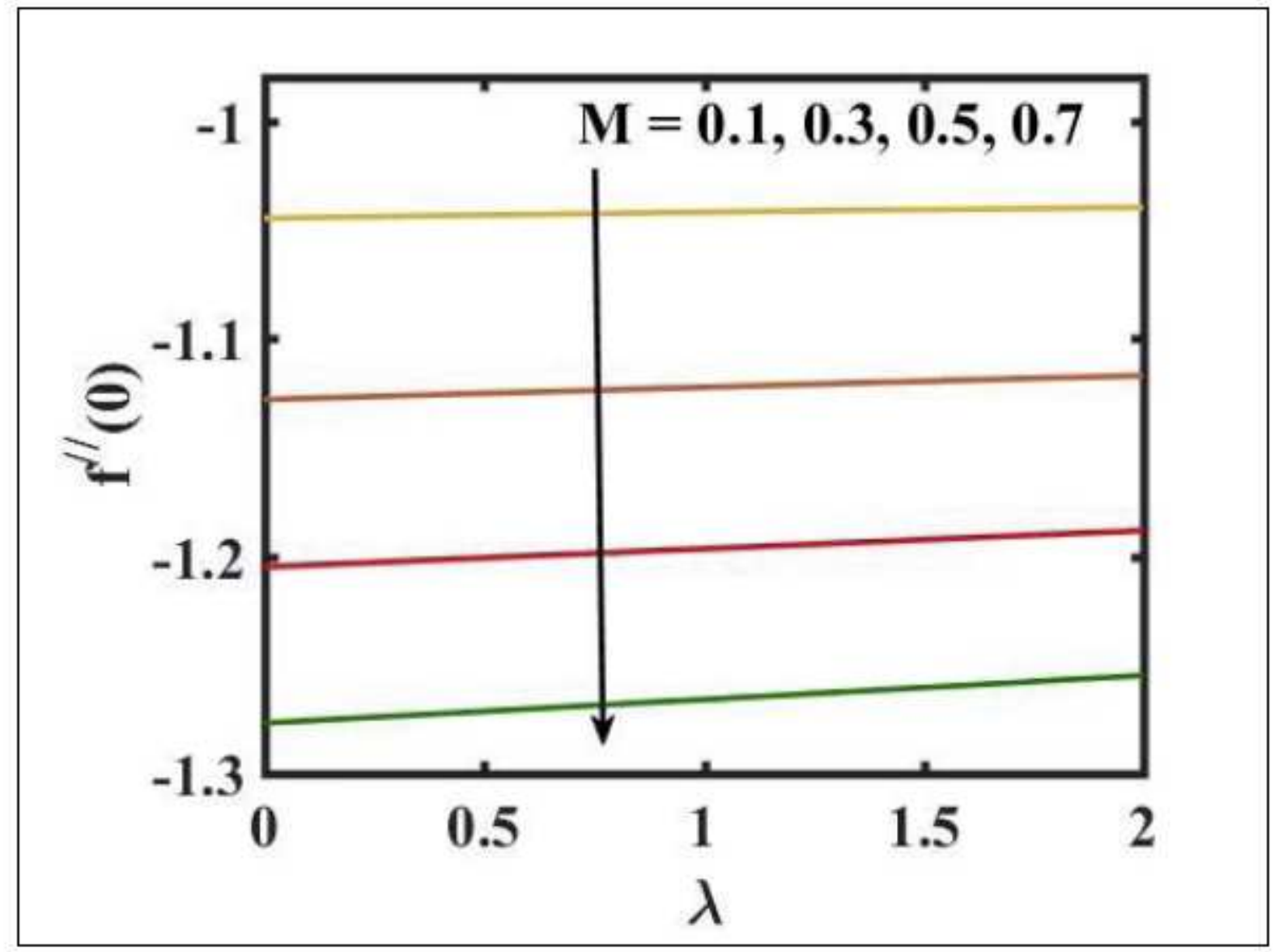

Figure 18

Encouragement of $M$ and $\lambda$. on skin friction coefficient 


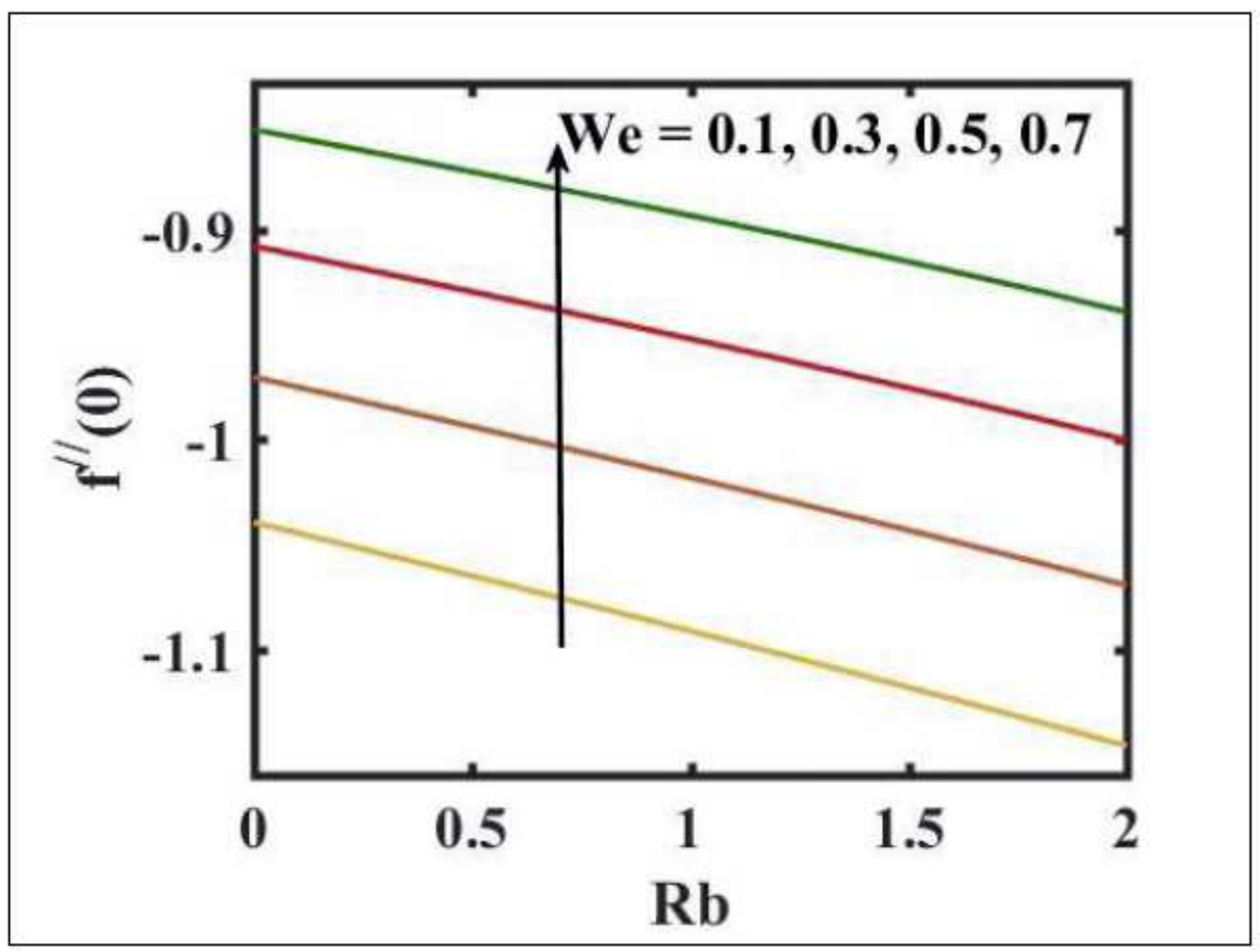

Figure 19

Encouragement We and $\mathrm{Rb}$ on skin friction coefficient 


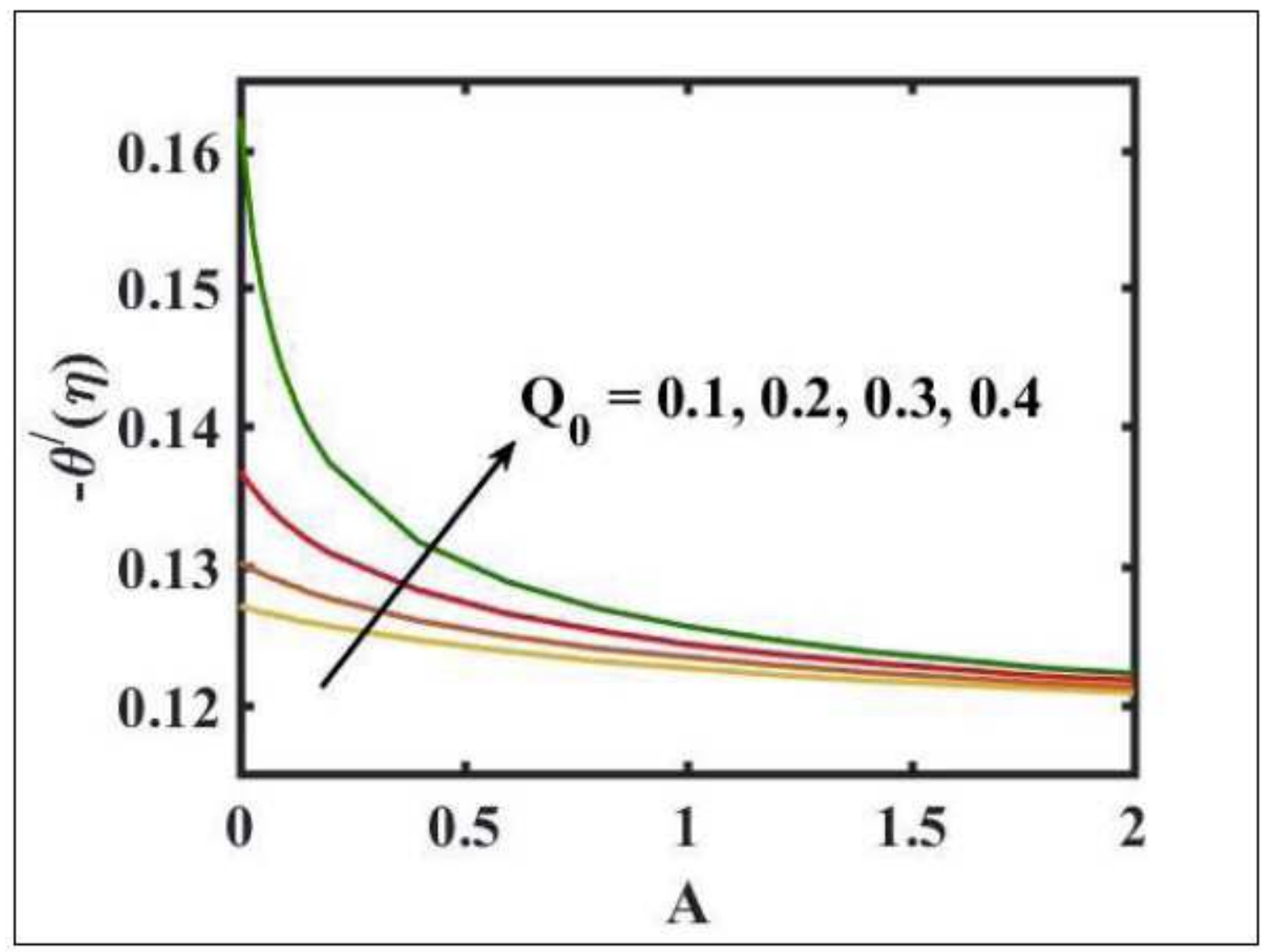

Figure 20

Encouragement of Q0 and A on local Nusselt number 


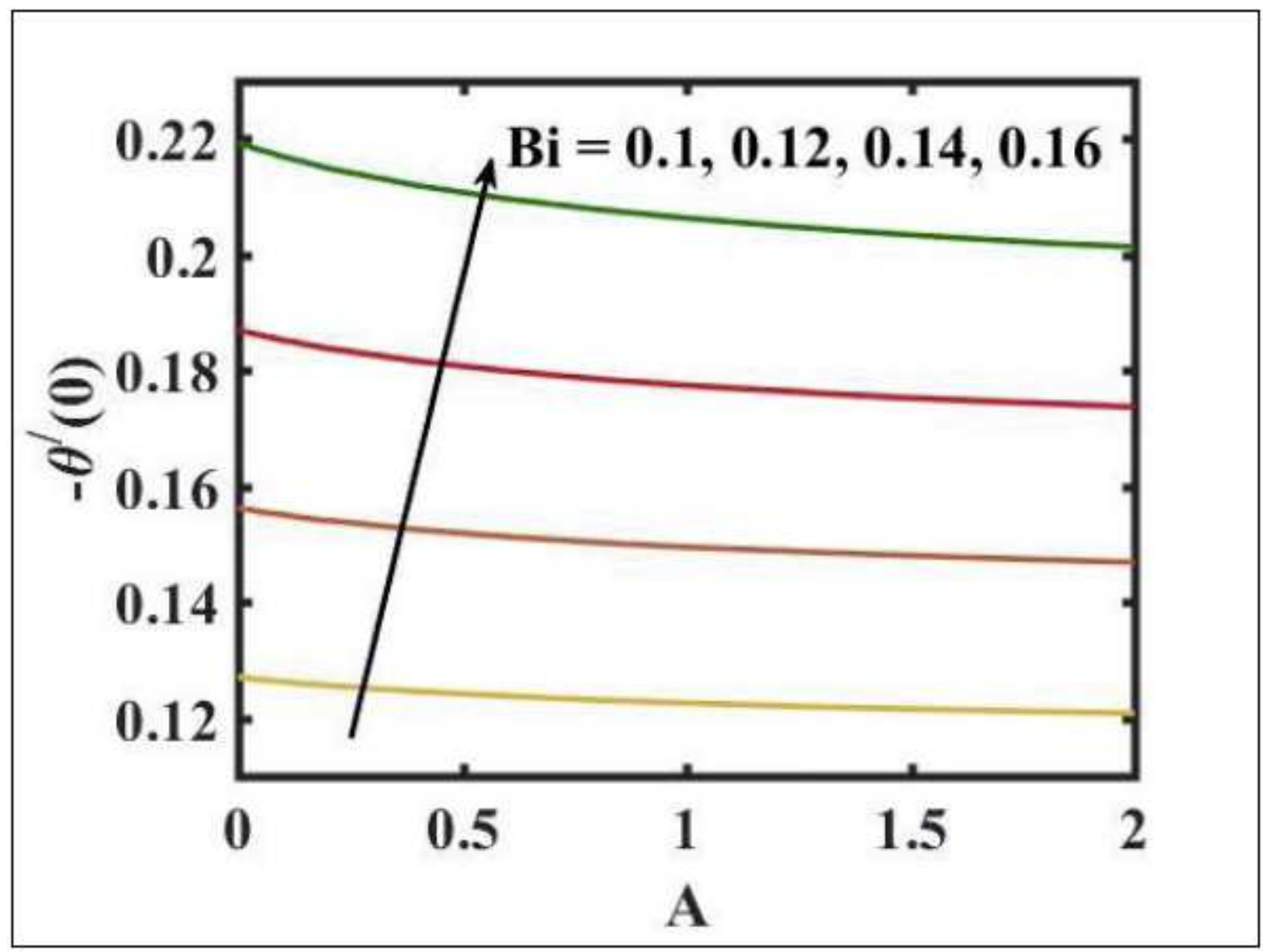

Figure 21

Encouragement of Bi and A local Nusselt number 


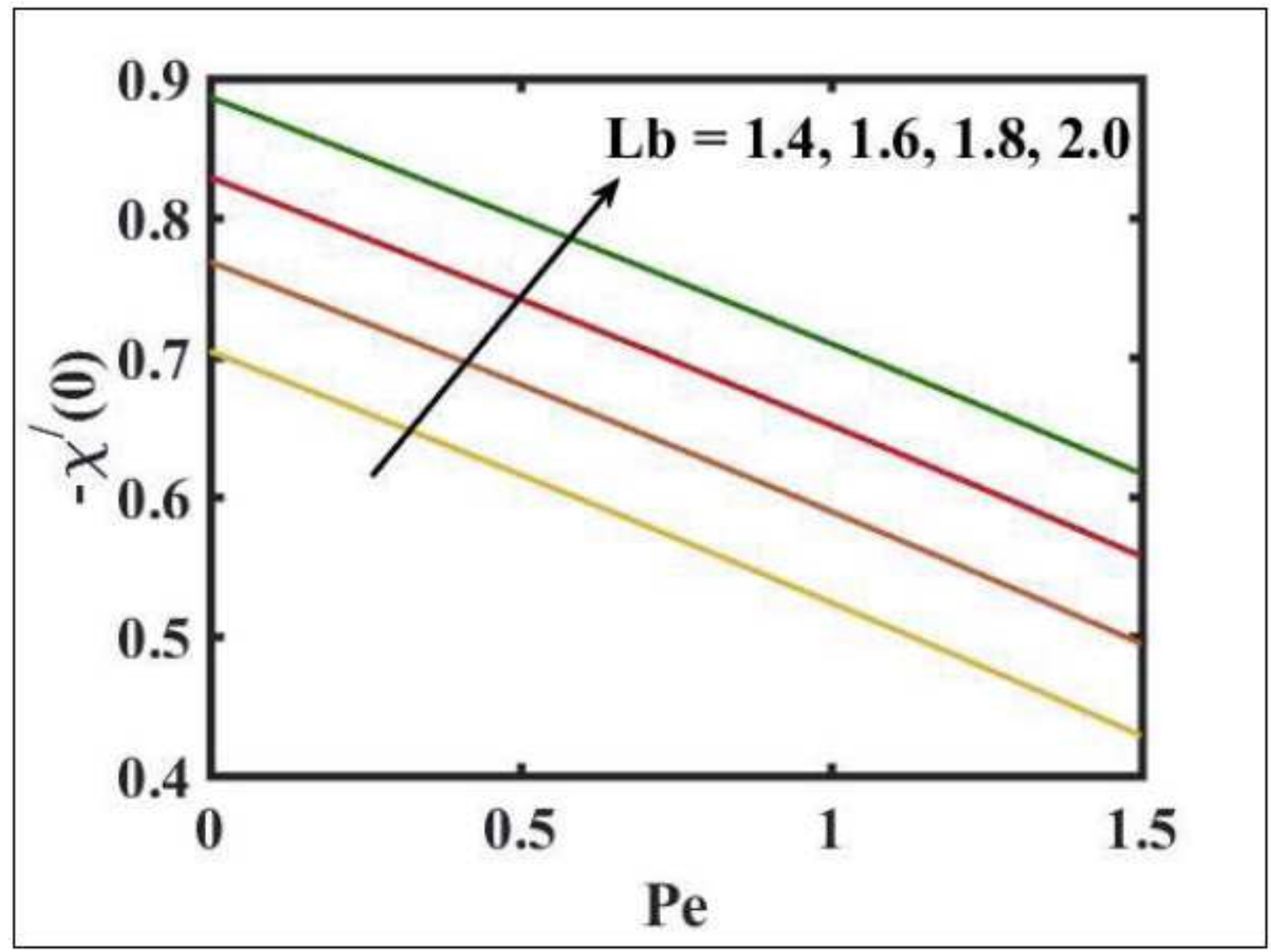

Figure 22

Encouragement of $\mathrm{Lb}$ and $\mathrm{Pe}$ on local density number of motile microorganisms 